Review

\title{
Metabolic and Microbial Modulation of the Large Intestine Ecosystem by Non-Absorbed Diet Phenolic Compounds: A Review
}

\author{
Juana I. Mosele, Alba Macià and Maria-José Motilva * \\ Food Technology Department, Agrotecnio Research Center, University of Lleida, \\ Av/Alcalde Rovira Roure 191, 25198-Lleida, Spain; E-Mails: juana.mosele@tecal.udl.cat (J.I.M.); \\ albamacia@tecal.udl.cat (A.M.) \\ * Author to whom correspondence should be addressed; E-Mail: motilva@tecal.udl.es; \\ Tel.: +34-973-702817; Fax: +34-973-702596.
}

Academic Editor: Carlo Siciliano

Received: 4 August 2015 / Accepted: 11 September 2015 / Published: 18 September 2015

\begin{abstract}
Phenolic compounds represent a diverse group of phytochemicals whose intake is associated with a wide spectrum of health benefits. As consequence of their low bioavailability, most of them reach the large intestine where, mediated by the action of local microbiota, a series of related microbial metabolites are accumulated. In the present review, gut microbial transformations of non-absorbed phenolic compounds are summarized. Several studies have reached a general consensus that unbalanced diets are associated with undesirable changes in gut metabolism that could be detrimental to intestinal health. In terms of explaining the possible effects of non-absorbed phenolic compounds, we have also gathered information regarded their influence on the local metabolism. For this purpose, a number of issues are discussed. Firstly, we consider the possible implications of phenolic compounds in the metabolism of colonic products, such as short chain fatty acids (SCFA), sterols (cholesterol and bile acids), and microbial products of non-absorbed proteins. Due to their being recognized as affective antioxidant and anti-inflammatory agents, the ability of phenolic compounds to counteract or suppress pro-oxidant and/or pro-inflammatory responses, triggered by bowel diseases, is also presented. The modulation of gut microbiota through dietetic maneuvers including phenolic compounds is also commented on. Although the available data seems to assume positive effects in terms of gut health protection, it is still insufficient for solid conclusions to be extracted, basically due to the lack of human trials to confirm the results obtained by
\end{abstract}


the in vitro and animal studies. We consider that more emphasis should be focused on the study of phenolic compounds, particularly in their microbial metabolites, and their power to influence different aspects of gut health.

Keywords: colon metabolites; gut fermentation; microbiota; phenolic compounds

\section{Introduction}

Most of the beneficial health properties of fruit, vegetables, and whole grains have been attributed to bioactive non-nutritional chemical compounds commonly named phytochemicals, which include phenolic compounds. Plant phenols embrace a wide range of secondary metabolites that are synthesized from carbohydrates via the shikimate pathway, occurring as soluble conjugated (glycosides) and insoluble or bound forms [1]. Based on the extensive intake of these kinds of phytochemicals through the diet, a complex mixture of hundreds of phenolic compounds enters the gastrointestinal tract where they can be partially released and absorbed, or survive stomach and intestinal digestion and reach the colon until excretion via feces. Several studies have reported that an important part of the ingested phenolic compounds reaches the large intestine where it undergoes a series of microbial transformations that leads to the generation of related metabolites [2-4].

On occasion, gut microbiota has been defined as a biological reactor since it possesses powerful metabolic functions which include the transformation of many compounds that reach the colon. This activity is possible through the capacity of microorganisms to produce a huge and varied range of enzymes. In the particular case of phenolic compounds, their intestinal transformations include several steps. First, aglycones must be released to the media. For this, different classes of enzymes are needed to deconjugate the specific moiety associated to the molecule or, in the case of polymeric forms, to break phenolic polymers into individual monomers. Phenolic compounds are also strongly linked to some components of the food matrix, and this interaction is also disrupted by microbiota [1]. The released aglycones undergo subsequent microbial transformations which may include ring fission, $\alpha$ or $\beta$-oxidation, dehydrogenation, dehydroxylation, and demethylation, and these result in the generation of simpler related compounds.

An overview of the bioactivity that is probably carried out by phenolic compounds in the large intestine is represented in Figure 1. The passage of digesta through the small intestine is estimated to take around 2-4 h, but the transit time increases considerably in the large intestine, extending to as long as $24 \mathrm{~h}$ or more $[4,5]$. This time is probably long enough to accumulate substantial amounts of phenolic compounds and induce metabolic and microbial changes in the gut lumen. Phenolic compounds are recognized antioxidants and anti-inflammatory agents which can protect intestinal cells from pro-oxidant and inflammatory injuries [6-8]. The presence of luminal phenolic compounds could also impact the metabolic profile, enhancing or inhibiting the generation of fermentation products derived from endogenous and dietary compounds. In turn, changes in the metabolic profile of the gut are sometimes associated with the modification of the shape of the intestinal inhabitants. In fact, some authors have associated the phenolic compounds-gut microbiota interaction with a presumable modulation effect, which could prevent or, indeed, restore microbiota alterations observed in disease $[9,10]$. 


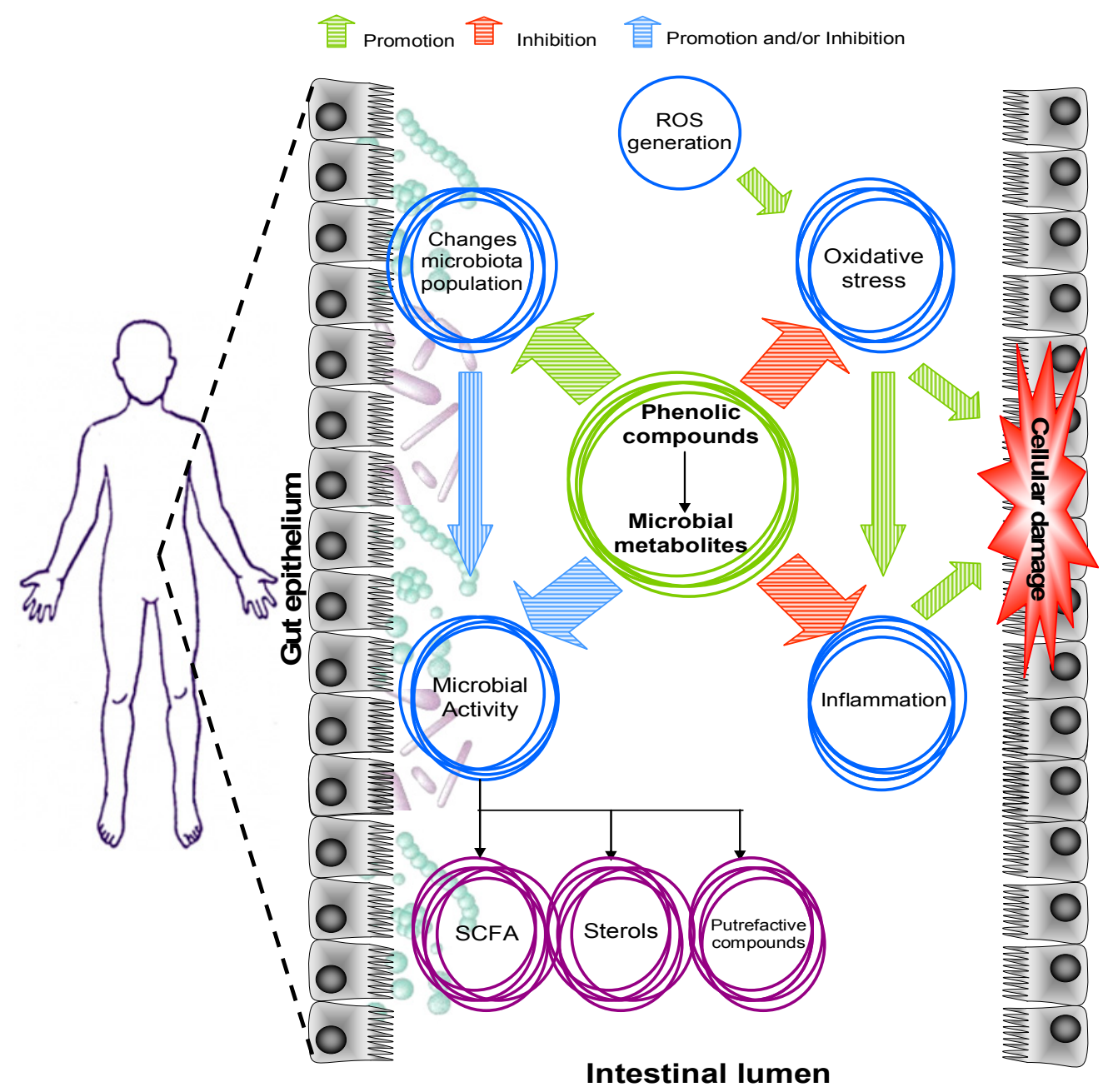

Figure 1. Overview of the possible implications of phenolic compounds at the gut level.

The aim of this manuscript was to consult the information available from in vitro and in vivo studies to review the transformations that the non-digested phenolic compounds undergo when they reach the colon and the possible influence they have on the transformation of other intestinal metabolites, such as short chain fatty acids (SCFAs), sterols, and microbial products of non-absorbed proteins. In addition, their possible implications in metabolic and microbial changes as well as in antioxidant and anti-inflammatory effects at the gut level were also considered.

\section{Microbial Phenolic Metabolites}

Phenolic compounds are commonly classified into two main groups, flavonoids and non-flavonoids. In the next section we will describe the catabolism pathways and the phenolic metabolites formed related with the chemical structures of flavonoids and non-flavonoids.

\subsection{Flavonoids}

Flavonoids possess an aglycone skeleton of two flavonoid rings (A- and B-rings) connected by a heterocyclic C-ring. In foods, except for flavan-3-ols, flavonoids are normally presented as glycosides or organic acid conjugates. 
The sub-class of flavan-3-ols includes the diastereomers catechin and epicatechin, and their corresponding gallate esters, epigallocatechin and epigallocatechin gallate. In foods, flavan-3-ols are present as monomers (catechin and epicatechin) or proanthocyanidins (variable attached monomers). To study the microbial modifications of flavan-3-ols, pure standards as well as grapes, tea, cocoa, berries, red wine, and their extracts have been used. The colonic catabolism of proanthocyanins undergoes a first degradation step resulting in the release of monomer structures with the subsequent hydrolysis of gallic acid from the galloylated forms [11-14]. Based on the literature, we propose the colonic pathways of monomeric flavan-3-ols and their corresponding gallate esters (Figure 2). The early appearance of simple phenolics (like catechol) suggests further degradation of gallic acid generated by hydrolysis of epigallocatechin gallate in the first steps of the colonic catabolism $[11,12,14,15]$. Similarly, the initial C-ring fission of flavan-3-ols (Figure 2A) produces the corresponding

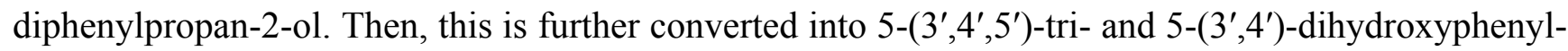
$\gamma$-valerolactone in the cases of gallate esters and monomers, respectively $[11,14,16]$. The subsequent catabolism of the tri- and dihydroxyphenyl- $\gamma$-valerolactones originates different hydroxylated forms of phenylvaleric acid [11-15,17]. Neither trihydroxyphenylvaleric acid nor trihydroxyphenylpropionic acid have been described as microbial metabolites of gallate esters in in vitro fermentations. However, trihydroxyphenylvaleric acid was identified in human plasma [16]. Phenyl- $\gamma$-valerolactones and phenylvaleric acids have been described as exclusive microbial metabolites of flavan-3-ols. Their progressive microbial transformation releases different hydroxylated forms of phenyl (Figure 2B) and benzoic acids (Figure 2C), whose profile and abundance seem to depend on the particular metabolic activity of each individual microbiota and the composition of flavan-3-ols in the substrate [11-15,17]. Furthermore, other minor catabolites, such as hippuric acid, $p$-coumaric acid, and vanillic acid, homovanillic acid, homovanillyl alcohol, and 3-O-methylgallic acid have been associated with the in vivo colon metabolism of flavan-3-ols $[11,13,16,18]$.

Flavonols are the most common phenolic compounds in foods such as red wine, apples, onions, beer, spices, herbs, berries, and cocoa, with quercetin, kaempferol, and myricetin being the most studied. During the first steps of the colonic catabolism of the aglycone forms of quercetin and kaempferol, the initial metabolites formed are dihydroquercetin (taxifolin) and dihydrokaempferol, respectively, which are further metabolized to di- and hydroxyphenylpropionic acid, respectively (Figure 3A) [19-23]. These metabolites then enter the catabolic route of phenyl acids (Figure 2B) and benzoic acids (Figure 2C) to generate minor related compounds [19,22,23]. Following the intake of radiolabeled quercetin-4'-O-glucoside in rats, high concentrations of hippuric acid were found in the jejunum/ileum, urine, and plasma [22]. Furthermore, 3',4',5'-trihydroxyphenylacetic acid and 3',5'-dihydroxyphenylacetic acid were the main microbial metabolites detected in rat feces after the administration of myricetin [24], probably derived from the intermediate dyhydromyricetin. However, the generation of quercetin from myricetin should not be discarded [25]. 
A.Flavan-3-ols pathway

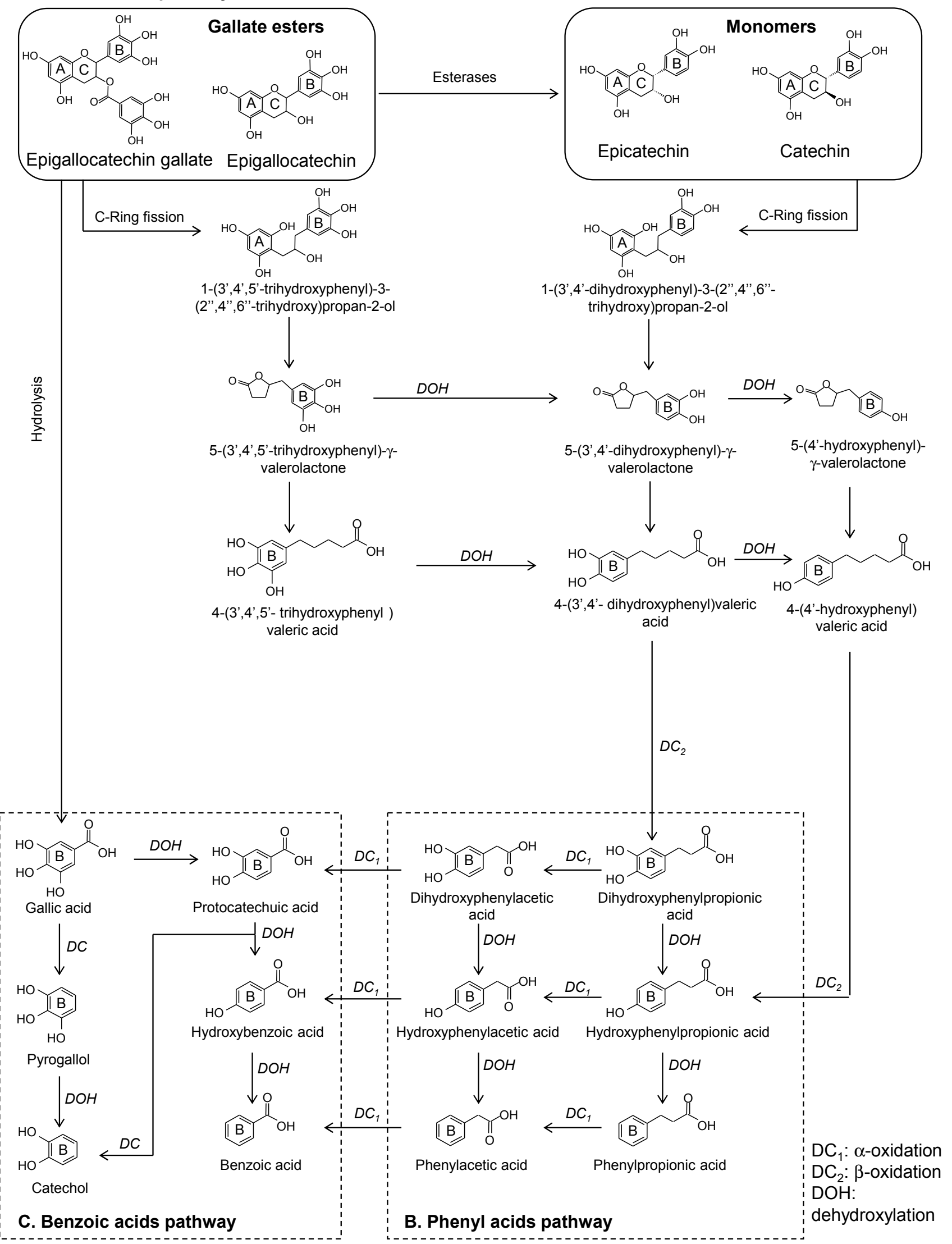

Figure 2. Proposed colonic pathways of monomeric flavan-3-ols and their corresponding gallate esters. 


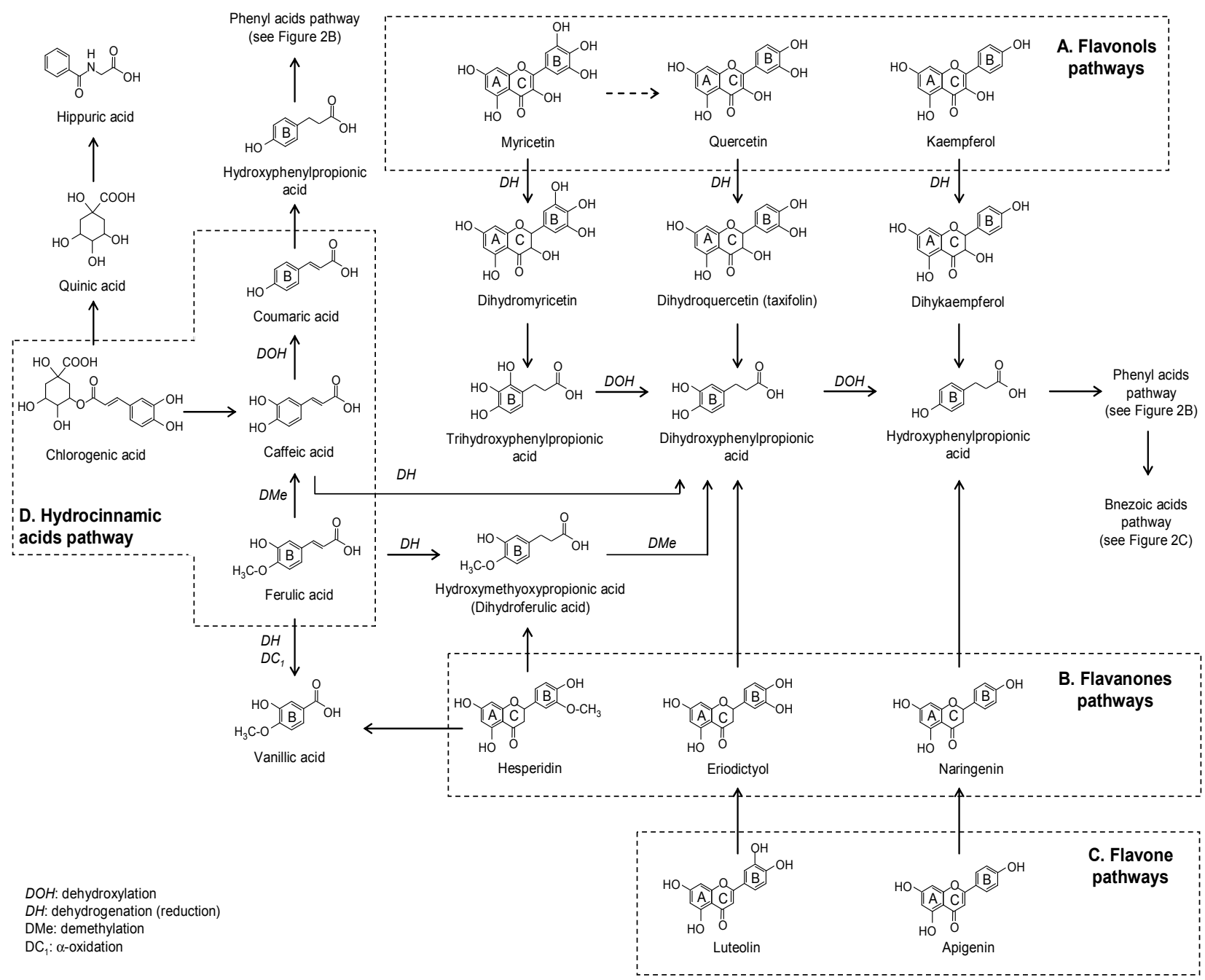

Figure 3. Proposed colonic pathways of flavonols, flavanones, flavones, and hydrocinnamic acids.

The flavanones hesperetin and naringenin are found in larger concentration in oranges, whereas eriodictyol is a flavanone commonly presented in aromatic herbs and nuts. Hydroxyphenylpropionic and phenylpropionic acids have been described as major fermentation products of eriodictyol and naringenin [23,26,27]. In addition, phloroglucynol was also detected in the fermentation vessels of naringenin [23] and eriodyctiol [28] which could be formed from the A-ring. In the case of hesperidin, 3-(3-hydroxy-4-methoxyphenyl)propionic acid (dihydroisoferulic acid) and different hydroxylated forms of phenylpropionic acid have been reported as products of it colonic catabolism [27] (Figure 3B).

Flavones, like apigenin and luteolin and their glycosides, are normally found in beer, olive oil, aromatic herbs, and nuts. In the same way as with the other flavonoid sub-classes, deglycosylation is the first microbial action. Apigenin aglycone is transformed into naringenin, whose microbial catabolism produces phenylpropionic acids [21,29] (Figure 3C). Luteolin undergoes an initial isomerization to form eriodictyol as an intermediate metabolite which is transformed to 3-(3',4'-dihydroxypheyl)propionic acid [21,30] (Figure 3C). In coincidence with in vitro data, 3-(4'-hydroxyphenyl)propionic acid was the main metabolite detected in urine after the administration of apigenin-7-O-glucoside to human microbiota-associated rats [29]. 
Genistein and daidzein are the most representative isoflavones and are normally present in their glycoside forms in soy products. The results of in vitro incubations of genistein, together with experiments where gnotobiotic rats were inoculated with isolated human equol-forming bacteria, have shown that, during the first steps of colonic catabolism, genistein, aglycone generated dihydrogenistein (Figure 4) which is partly converted into 5-hydroxy-equol [31,32]. After in vitro fermentation of genistein, Coldham et al. [33] observed that dihydrogenistein was metabolized into 6'-hydroxy-Odemethylangolensin which, in turn, was transformed into 2-(4-hydroxyphenyl)propionic acid and phloroglucinol. On the other hand, daidzein aglycone can be reduced to dihydrodaidzein and then converted into equol or $O$-desmethylangolensin [31,32,34] (Figure 4). Although more than one bacteria has been described as an isoflavone-converter [31,32], not all humans have the capacity to convert genistein and daidzein into their respective microbial metabolites [35], which could indicate that isoflavone transformer bacteria are not common among human intestinal flora.

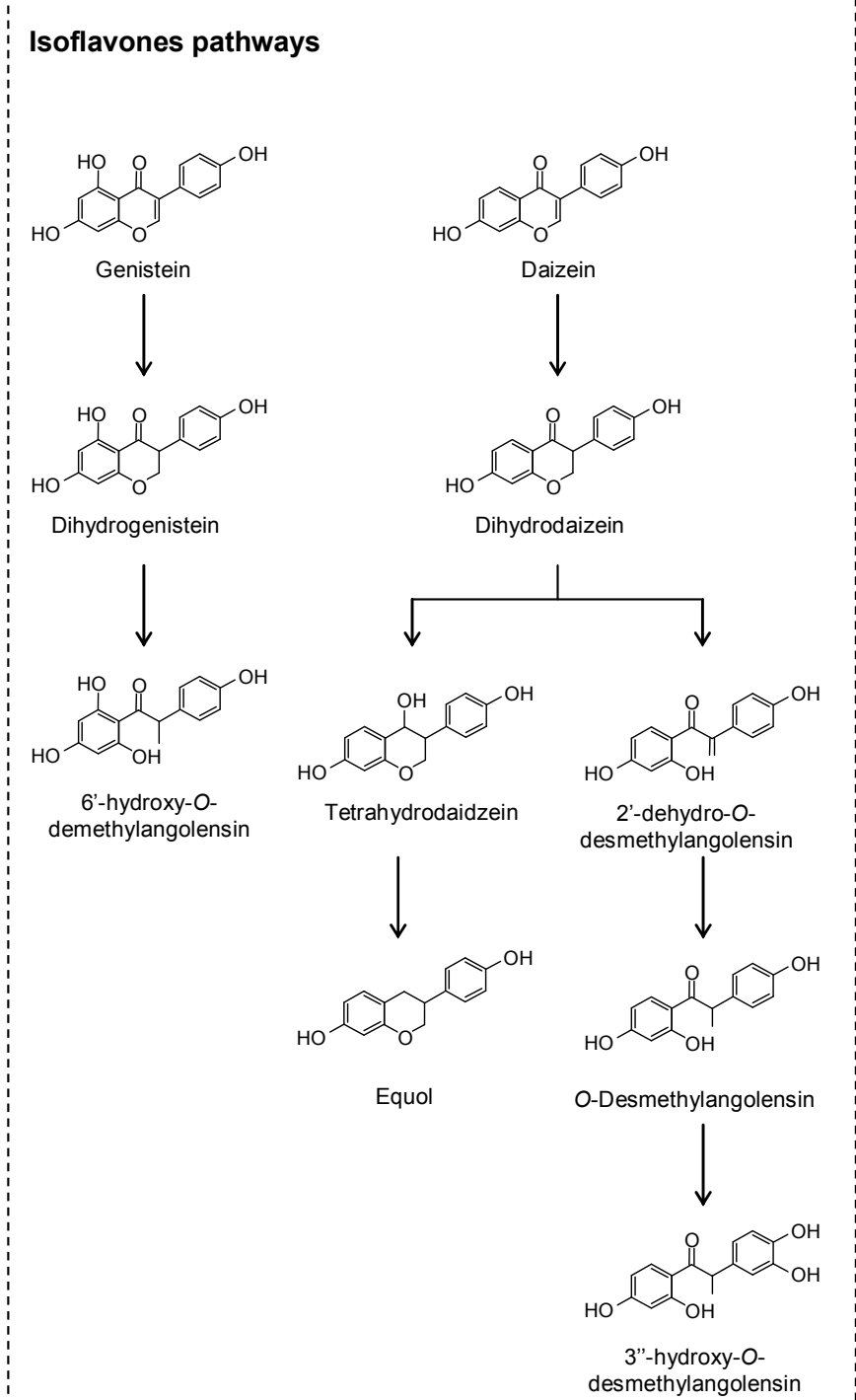

Figure 4. Proposed colonic pathways of isoflavones.

Anthocyanins are widely dispersed throughout the plant kingdom, being responsible for red, blue, and purple colors. Red wine, grapes, berries, and pomegranate are examples of anthocyanin-rich 
products. After microbial deglycosylation, ring fission of the aglycone releases two parts, one from the A-ring and the second one from the B-ring, which then undergo simultaneous catabolism [36,37]. Regarding the fission of the B-ring, both phenyl acids and benzoic acids have been reported as the microbial metabolites of anthocyanins (Figure 5). Gonzalez-Barrio et al. [37] proposed a complete colonic pathway for cyanidin including different alternative conversion pathways for the B-ring, and protocatechuic acid (benzoic acid) and 3-(3',4')-dihydroxyphenylpropionic acid (phenyl acid) were proposed as initial metabolites. The subsequent microbial metabolism of these initial products generates simpler compounds. Regarding benzoic acids, hydroxybenzoic acid has been reported to be a microbial metabolite of pelargonidin glucoside, protocatechuic acid of cyanidin glucoside, vanillic acid of peonidin glucoside, syringic acid of malvidin glucoside, methyl gallic acid of petunidin glucoside, and gallic acid of delphinidin glucoside [36-40]. Considering the results obtained from previous studies, the generation of benzoic acids prevails over that of phenyl acids (Figures 2B,C and 5).

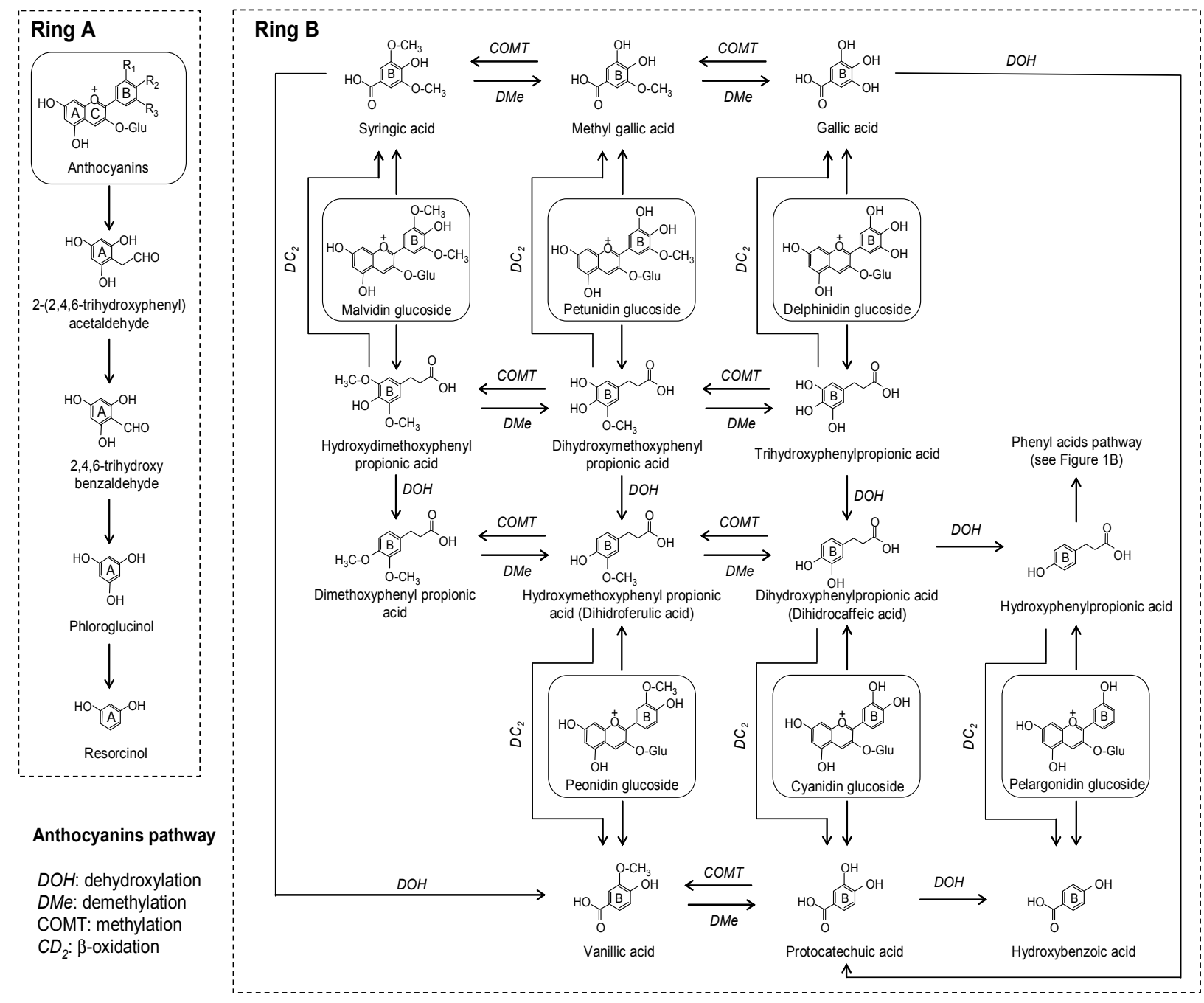

Figure 5. Proposed colonic pathways of anthocyanins.

Apart from B-ring fission, other microbial metabolites could also be generated from A-ring fission. The fission of ring A of anthocyanins could generate trihydroxybenzaldehyde [39,40], which could further be converted to phloroglucinol [37]. Due to the lack of complete information regarding the 
description of the colonic fate of some anthocyanins, we tentatively propose alternative pathways in Figure 5. Obviously, the acceptance or rejection of these must be confirmed by future studies. Along with some of the metabolites described in in vitro studies, hippuric acid was also detected in human urine after the ingestion of raspberries [37].

\subsection{No Flavonoids}

Phenolic acids, such as hydroxycinnamic and hydroxybenzoic acids, are present in numerous plant products. In plant tissues, phenolic acids form ether linkages with lignin through their hydroxyl groups in the aromatic ring and ester linkages with structural carbohydrates and proteins through their carboxylic group [1]. These bound phenolics survive stomach and intestinal digestion and reach the colon since, being the substrate to colon microbiota, cell wall fibrous materials are difficult to digest.

Hydroxycinnamic acids are widely distributed in nature, with coffee, whole cereals, dried drupes, wine, berries, spices, and aromatic herbs being the richest sources. Caffeic, ferulic, and $p$-coumaric acids, as well as their tartaric and quinate esters, such as chlorogenic acid (quinate ester of caffeic acid), are included in this group, and their microbial degradation steps are proposed in Figure 3D. Chlorogenic acid underwent dihydroxylation, dehydrogenation, or ester hydrolysis as a first microbial transformation in simulated conditions [41]. In vitro fermentation of freeze-dried coffee confirmed the initial hydrolysis of chlorogenic acid into caffeic acid which later suffers degradation, with di- and mono-hydroxylated phenylpropionic acids the main metabolites detected [23,42]. Other metabolites, such as $m$-coumaric and hippuric acids, were detected in the urine of rats after the administration of chlorogenic acid [43]. As an intermediate product of chlorogenic acid, caffeic acid degradation was expected to produce a similar metabolic profile [43] (Figure 3D). Dihydroferulic acid (3-(3-methoxy4-hydroxyphenyl)propionic acid) [27,39] and 4-vinylguaiacol [44] together with minor degradation compounds have been described after in vitro fermentation of ferulic acid.

On the other hand, hydroxybenzoic acids are made up of gallic acid and ellagic acid. Gallic acid is also part of the hydrolysable tannins' and flavan-3-ols' molecular structure. Its microbial transformation is proposed in Figure $2 \mathrm{~B}$, whereas the colonic fate of ellagic acid is explained in the following section.

Ellagitannins are the main group of hydrolysable tannins. Particularly high concentrations of ellagitannins are found in muscardine grapes, pomegranates, and some berries and nuts. Intestinal breakdown of ellagitannins into ellagic acid was observed in vitro [2,37,45] and in vivo [46]. Ellagic acid is further metabolized by local bacteria, giving pentahydroxy-urolithins as the first metabolite which is successively dehydroxylated to tetra-, tri-, di-, and mono-hydroxy-urolithins, [2,37,45-47], as proposed in Figure 6A. An important person-to-person variability in the profile and amounts of urolithins has been observed with major urolithin A (dihydroxy-urolithin) or urolithin B (hydroxy-urolithin) producers, and others are incapable of producing any class of urolithins [2,3,37,45,47]. Gordonibacter urolithinfaciens and Gordonibacter pamelaeae were described as responsible for the transformation of ellagic acid into penta-, tetra-, and trihydroxyurolithin [48] and its absence could be associated with the inability of some individuals to produce urolithins. 

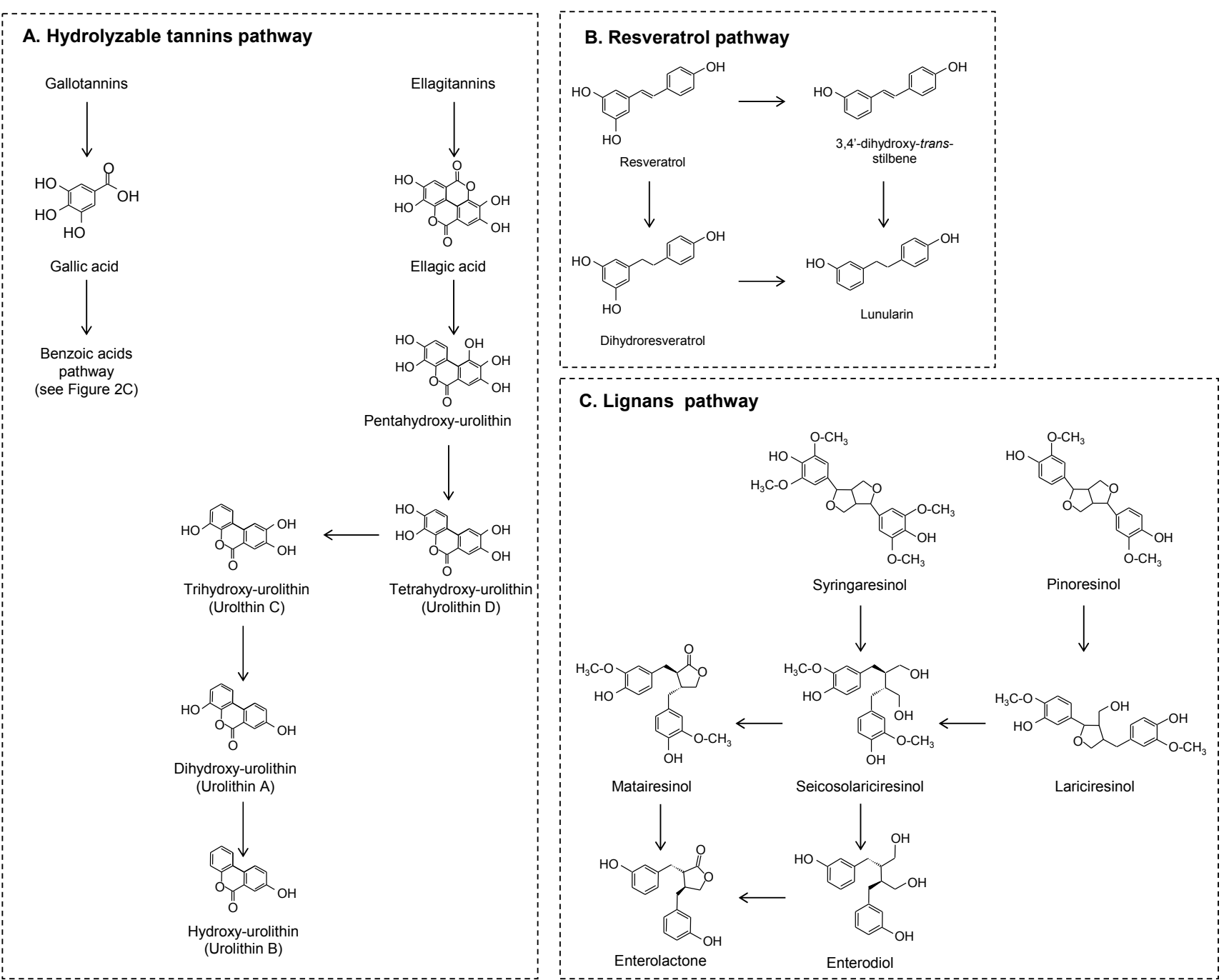

Figure 6. Proposed colonic pathways of hydrolysable tannins, resveratrol, and lignans.

Resveratrol (3,5,4'-trihydroxy-trans-stilbene) is the most common stilbene, present in grapes and wine, and it has been extensively studied. Regarding the in vitro experiments, dihydroresveratrol, 3,4'-dihydoxy-trans-stilbene, and lunularin (3,4'-dihydroxy-bibenzyl) were described as microbial derivatives [49,50] (Figure 6B). Bode et al. [49] concluded that three colonic pathways of resveratrol can be distinguished in terms of the quantity of end products as a lunularin producer, dihydroresveratrol/lunularin producer, or dihydroresveratrol producer. Whereas dihydroresveratrol and lunularin were considered the main end products, the low amounts of 3,4'-dihydroxy-trans-stilbene detected seem to indicate that this product is intermediate or marginally produced by gut microbiota. The urine metabolic profile after an acute dose of trans-resveratrol confirmed the results found under in vitro fermentation $[49,50]$.

Syringaresinol, pinoresinol, lariciresinol, secoisolariciresinol, and matairesinol are the most common lignans found in plant products. Besides being present in foods, secoisolariciresinol is a microbial degradation product of syringaresinol and also of lariciresinol, which, in turn, is derived from the microbial fermentation of pinoresinol [51,52] (Figure 6C). The final microbial products of secoisolariciresinol, with several intermediates, are enterodiol and its oxidized product, 
enterolactone [53,54]. Secoisolariciresinol can also be converted to matairesinol, from which only enterolactone is obtained from microbial catabolism [52].

The phenolic alcohols, tyrosol, hydroxytyrosol and its precursors, oleuropein, and hydroxytyrosol acetate are the most representative compounds typically found in olives and virgin olive oil. Few studies have focused on the colonic pathway of these phenolic compounds. Oleuropein was transformed in vitro into its aglycone, elenolic acid, hydroxytyrosol, and hydroxytyrosol acetate [55,56] (Figure 7). Individual fermentation of tyrosol and hydroxytyrosol confirmed the low microbial metabolism of these compounds and their relative stability under in vitro colonic conditions [55]. An in vivo experiment following the administration of oleuropein to rats detected other related metabolites such as homovanillic acid [57].

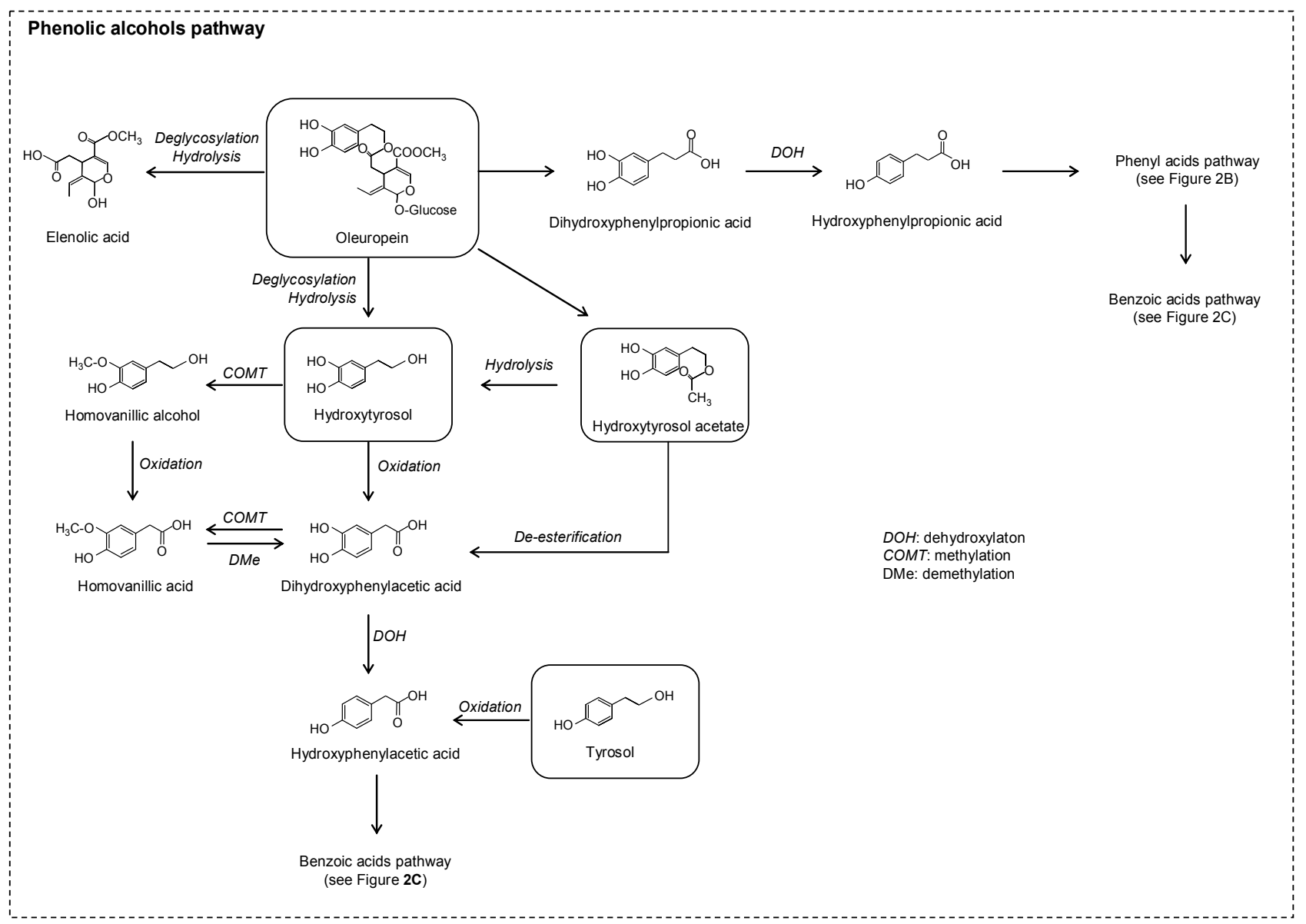

Figure 7. Proposed colonic pathways of oleuropein.

Considering the complex colonic pathways, it is possible to conclude that the first microbial transformation of phenolic compounds leads to the accumulation of initial metabolites that share characteristics from the original compound (particularly conserving their functional groups) and are considered markers of an early fermentation stage. The continuous exposure of the initial metabolites to microbiota leads to the accumulation of final catabolites, which can be common to different phenolic sub-groups. An elevated accumulation of some microbial metabolites would be indicative of the main microbial pathways probably common to several individuals. On the contrary, those 
metabolites present in minor concentrations or in a smaller proportion of individuals could be related to a specific microbiota composition capable of activating secondary metabolic pathways.

Different profiles of metabolites observed after in vivo interventions suggest hepatic and renal metabolism prior to excretion via urine. Hippuric acid was the common phenolic metabolite detected in the largest amounts in plasma and urine after the intake of varied phenolic sources. The origin of hippuric acid can be through the microbial transformation of the quinic acid moiety or the hepatic metabolism of benzoic acids [58,59]. Nevertheless, the concentration of hippuric acid in urine decreased considerably after the antibiotic treatment of rats [60] and in the urine of ileostomy [11], suggesting an important contribution by the gut microbiota.

\section{Box 1. Models Concerning the Study of Phenolic Microbial Metabolites}

In vitro and in vivo studies have been essential for building the colonic pathways of phenolic compounds. Basically, in vitro studies consist of anaerobic incubations where phenolic compounds represent the microbial substrate and bacteria cultures, human or animal feces, the microbial inoculum. The phenolic substrate used in these studies may include pure standards $[11,19,20,26,30,38,49,55]$, isolated compounds [36,38,45], phenolic extracts $[12,15,38,39,45]$, and food or pre-digested food [2,37,42,57]. In vitro fermentation models have some questionable points, mainly their limited representativeness of in vivo conditions. For example, in vitro incubations do not include enterohepatic recirculation, colonocyte absorption, mucosa-associated microbiota, and changes in physiological conditions during the transit time. The latter point has been partly overcome with the development of modern controlled batch cultures called "Simulator of Human Intestinal Microbial Ecosystem" (SHIME). The sample circulates through different reactors that represent the ascending, transversal, and descending colon in which $\mathrm{pH}$ and temperature are continuously controlled and the growth of microbiota is in line with the environmental conditions and substrate availability [12,17,61]. Despite the mentioned limitations, in vitro studies provide valuable information regarding the phenolic metabolites generated by gut microbiota. Fermentations are usually performed for $24-48 \mathrm{~h}$ during which sub-samples are collected at different time-points. Time-course metabolite generation allows colon metabolic pathways that probably take place under in vivo conditions to be defined. As microbial metabolites can be absorbed by colonocytes, their in vitro identification provides a useful tool for identifying these phenolic metabolites in plasma and/or urine in the human bioavailability or dietary intervention studies.

In vivo studies are ideal complements to the in vitro ones. Nevertheless, complex ethical issues, concerning the study of the phenomena occurring during human digestion, limit in situ observations and sample collection. In general, phenolic compounds are usually analyzed in fractionated blood (plasma, serum...) and urine to determine which compounds are absorbed and their absorption and excretion kinetics. In general, phenolic compounds are quickly absorbed, reaching the $\mathrm{C}_{\max }$ in plasma between 0.5 and four hours after the intake, corresponding to the stomach and/or small intestine absorption [16,18,37,62], whereas the colonic metabolites appear in plasma later, indicating their gradual colonic biotransformation and, in some cases, showing a second increase in the plasma concentration after $4 \mathrm{~h}[16,18]$. 
In vitro and in vivo studies have contributed enough so far to confirm the capacity of gut microbiota to metabolize phenolic compounds (Box 1). The degradation pathway routes involve several reactions in which the side chain groups and the heterocyclic C-ring of flavonoids are more likely to be used by microorganisms, while the benzoic ring remains intact. Depletion of phenolic compounds along with the increase in bacteria growth observed in in vitro fermentations could be considered good evidence to believe that these phytochemicals are used as carbon and energy sources. However, there are other reasons that can explain the utilization of phenolic compounds by gut microbiota. As xenobiotics, they may be degraded to reduce the toxicity that these compounds may have on resident bacteria.

\section{Impact of Diet Phenolic Compounds on Gut Microbial Fermentation Metabolites}

\subsection{Short Chain Fatty Acids (SCFAs)}

SCFAs are saturated aliphatic organic acids consisting of one to six carbons, of which acetic (C2), propionic (C3), and butyric (C4) acids are the most abundant. SCFAs production mainly occurs in the proximal part of the colon, where the availability of substrates is most abundant. The majority of SCFAs (up to 95\%) are rapidly absorbed by the colonocytes, leading to decreasing concentrations from the proximal to distal colon. Only a minor fraction of the SCFAs (about 5\%) is excreted in the feces [63]. Isobutyric, isovaleric, and valeric acids, also called branched chain fatty acids (BCFAs), constitute the remaining 5\%-and are derived from the microbial fermentation of branched chain amino acids $[64,65]$. A disruption in the concentration of SCFAs, especially due to an increase in the proportion of BCFAs, could be a possible signal of loss of intestinal homeostasis $[66,67]$.

Acetic acid, propionic acid, and particularly butyric acid have been extensively studied due to their involvement in the maintenance of correct body functions [68,69]. Butyric acid is considered essential for maintaining the colon cellular function since it is the main energy source for colonocytes [70]. Chemopreventive properties have also been described for butyric acid due to its capacity to prevent the formation of malignant cells and to induce apoptosis in colonic cancer cells [71]. In fact, lower amounts of SCFAs [72] and butyric acid-producing bacteria [73] were found in the feces of colorectal patients than in those of healthy subjects. Contrarily, elevated amounts of BCFAs are presumed to be prejudicial to colonic health [74,75] and high concentrations have been observed in inflammatory bowel diseases (IBDs) [66] and obesity [67].

In vitro fermentation of pure phenolic compounds enables the extent to which phenolic compounds and gut microbiota are exclusively involved in SCFAs production to be seen. An improvement in the total production of SCFAs was observed after the in vitro incubation of chlorogenic acid, rutin, caffeic acid, quercetin [76], and olive oil polyphenols [77]. To the contrary, isolated proanthocyanidins [78] and punicalagins [45] suppressed the in vitro generation of SCFAs. The increments observed leave interpretations open to the thought that phenolic compounds could be transformed into SCFAs. However, this alternative is practically discarded since the ring cleavage of the aromatic ring of phenolic compounds was not observed under anaerobic conditions [79]. Rather than direct production, the increase in SCFAs observed after in vitro fermentation of pure phenolic standards is probably related to a higher fermentation rate of the released glycoside moieties and/or of the remaining carbohydrates in the culture media or the feces. 
Phenolic-rich extracts have increased in interest since they can provide added value as food ingredients and can also be used in nutraceutical applications. Their involvement in SCFAs generation has shown mixed results. In two experiments, black tea extract, red wine-grape juice extract [17], and soy germ powder [61] were studied in a SHIME in vitro system. The tea extract and soy germ powder increased the concentration of acetic and propionic acids, whereas the soy germ and the mix extract only increased the concentration of propionic acid. In parallel, a slight decrease in the amount of butyric acid was observed after continuous feeding of black tea and red wine-grape juice extracts. A rise in the production of the three main SCFAs was observed after incubation of pomegranate extract [45]. Higher recoveries of urolithins in the batch cultures of pomegranate extract compared with that of punicalagins suggest major microbial activity and a possible involvement of phenolic microbial metabolites. Nevertheless, rats fed with raspberry seed extract produced more SCFAs and minor urolithins than rats that received strawberry seed extract [80]. Thus, the results of different studies are contradictory and phenolic compounds with similar chemical characteristics showed different behavior.

Because the later data was obtained under in vitro conditions, it risky to extrapolate the same effects to in vivo conditions when extracts or phenolic compounds are ingested through the diet. Heterogeneous data have also been obtained from animal and human studies after exposure to a phenolic-rich diet. The concentration of acetic acid increased in the feces of pigs after two weeks of receiving a diet enriched with $0.2 \%$ of green tea phenolic compounds [81]. In healthy humans, the inclusion of a red wine-grape juice extract, but not grape juice extract, over four weeks, reduced the concentration of isobutyric acid [82]. Furthermore, a decrease in the production of SCFAs in rats was noticed after the administration of an aqueous passion fruit leaf extract [83]. No changes in the production of SCFAs were observed after the inclusion of trans-resveratrol or quercetin (alone or in combination) in rats fed with a high-fat sucrose diet for six weeks [84] or in healthy humans after the intake of pomegranate juice for four weeks [85].

The role of non-digestible carbohydrates (dietary fiber) in the production of SCFAs is unquestionable. Based on its chemical and physical properties, dietary fiber is normally classified into soluble and insoluble. Pectin, oligosaccharides (fructooligosaccharides, FOS), and inulin are considered soluble fiber. The fermentability of soluble fiber is high due to its capacity for water solubilization, which allows the action of microbial enzymes. Insoluble fiber corresponds basically to cellulose, some hemicelluloses, lignin, and arabinoxylans, which are also fermented, but at a much lower rate than soluble fiber [86]. Many authors have postulated that the production of SCFAs may be enhanced when dietary fiber is administered in combination with phenolic compounds. However, this claim remains inconclusive.

Replacement of part of the cellulose (maize starch) by strawberry and raspberry defatted seed extracts rich in ellagitannins enhanced the total SCFAs production and the proportion of butyric acid in rats after four weeks [80]. However, the results of the latter study were not only attributed to the phenolic compounds since their effectiveness was more marked in the raspberry extracts, coinciding with a major content of soluble fiber. In a similar experimental design, equivalent concentrations of dietary fiber were included in the diets of rats through soluble and insoluble fractions of bilberries, blackcurrants, and raspberries [87]. Rats fed with the soluble fraction of bilberries showed the highest caecal formation of SCFAs and fiber fermentability in coincidence with higher anthocyanin intake. 
Moreover, the addition of apple-pomace extract, in combination with FOS and cellulose, for four weeks [88], or lyophilized apple together with apple pectin [89], increased the proportion of butyric acid in rats compared with when fiber was consumed alone, suggesting an additional benefit that could be attributed to the presence of phenolic compounds in the extract.

In some cases, the combination of fiber with phenolic compounds seems not to alter the gut metabolism of SCFAs. For example, apple fiber rich in phenolic compounds did not produce any change in the generation of SCFAs, either in combination with apple pectin under in vitro conditions [71] or together with boysenberry juice after four weeks of human consumption [90]. In line with the previous studies, rats fed with apple-pomace extract rich in proanthocyanins showed no difference in the production of SCFAs compared with rats fed with apple-pomace extract deprived of phenolic compounds [91], and similar results were observed after combination of grape extract and inulin in the diet of rats [92]. Inhibitory effects in the production of SCFAs were observed when isolated apple polysaccharides were fermented in vitro together with purified apple phenolic extract rich in proanthocyanins [79]. Similarly, the suppression of SCFAs was also noticed in rats after four weeks of a combination of wheat and oat fiber with blackcurrant and chokeberry extracts (both rich in proanthocyanidins) [93] and low or high ellagitannin-rich strawberry extract in combination with FOS [94]. The conversion of SCFAs from starch was also suppressed by the addition of grape-seed extract to pig fecal incubations [95].

Since many factors such as the abundance and activity of specialized carbohydrate-fermented bacteria and enzyme expression and activity, as well as substrate availability, are involved in SCFAs synthesis, phenolic compounds could interact with one or more of these factors. Depending on the type and amount of the phenolic compounds ingested, they reach the colon in different proportions and degrees of polymerization [4,62]. In accordance with this, they could promote or inhibit the growth of carbohydrate-fermented bacteria [76,96]. Bacterial enzymatic expression and activity is crucial for the release of monomeric compounds from polysaccharides to form SCFAs. In addition, different works suggest that polyphenols might modulate the activity of glyosidic enzymes differently [80,92,93].

In plant foods, phenolic compounds are naturally associated with dietary fiber. Thus, the physiological effects of fiber may depend not only on its chemical and physical properties, but also on its particular linkage and the composition of its phenolic compounds [44,97]. The effects observed to be hampered, particularly by proanthocyanins, may be associated with tight bonds among fiber and phenolic compounds which result in a delay in degradation and, thus, less fermentation. Nevertheless, Snelders et al. [44] observed that fiber fermentability was more related to the amounts of phenolic compounds present in the fiber matrix than to their form (free or bonded to fiber). Thus, performing studies to define the intrinsic composition of dietary phenolics and food fiber could be of great interest for understanding the role of both food components when these are alone or together.

However, it is still questionable whether it is really desirable to stimulate the production of SCFAs. As SCFAs also represent energy extraction of non-absorbed macronutrients, they can provide approximately $10 \%$ of the total energy intake [98]. This aspect is considered positive in populations with a diet rich in plant-derived polysaccharide food. Nevertheless, this may be detrimental in Western populations, because a greater capacity to harvest energy from the diet could contribute to the positive calorie intake balance typical of obesity [99]. In fact, the gut microbiota of obese individuals are especially rich in bacteria specialized in carbohydrate fermentation $[99,100]$. Hindering of SCFAs 
production observed for some classes of phenolic compounds may be effective in reducing the energy harvest in overweight and obesity, which might partially explain the weight loss observed after the intake of food rich in phenolic compounds [101]. In synthesis, the versatility of phenolic compounds and their association with fiber, free or bound, could stimulate the design of different food products or recommendations depending on the effects expected to be attained.

\subsection{Bile Acids and Sterols}

Bile acids (BAs) are classified into primary and secondary. In humans, the primary BAs cholic and chenodeoxycholic acids are synthesized from cholesterol by hepatic enzymes and stored in the gallbladder (conjugated with the amino acids glycine and taurine) until being excreted into the duodenum to facilitate the digestion and absorption of lipophilic compounds. Most of the conjugated primary BAs are reabsorbed in the ileum and the remaining fraction reaches the colon, where cholic and chenodeoxycholic acids are metabolized into deoxycholic and lithocholic acids, respectively, by gut microbiota [102]. The main microbial catabolism steps include the deconjugation of amino acids and dihydroxylation by microbial 7 - $\alpha$-dehydroxylase $[102,103]$.

Fecal cholesterol represents the non-absorbed dietary cholesterol in addition to that provided by intestinal desquamated cells. The microbial reduction of cholesterol releases coprostanol as the main metabolite, generating cholestanone and coprostanone as intermediates [104]. High excretion of sterols and their microbial metabolites through feces has been associated with hipocholesterolemic effects [105], but is also a common feature of high fat diets [106]. A continuous exposure to elevated luminal levels of secondary BAs and cholesterol microbial metabolites has been suggested to increase the susceptibility to intestinal inflammation and colorectal cancer [74,107-109]. In addition to the toxic effects of secondary sterols per se, the concentration of putrefactive compounds (see Section 3.3 below) could also increase the lumen toxicity due to the release of amino acids (glycine and taurine), these being products of the microbial hydrolysis of BAs.

There is scarce data regarding the impact of phenolic compounds on the gut microbial transformation of BAs and cholesterol. Different studies have demonstrated that phenolic compounds have suppressing effects on the microbial conversion of sterols. For example, the amount of secondary BAs was reduced in rats fed with a diet containing tea polyphenols and gallotannins [110] or apple, grape, and red beet juices [111]. In another study with rats, the supplementation of a high fat diet with curcumin and caffeic acid reduced the concentration of deoxycholic acid in feces, whereas the administration of caffeic acid, catechin, rutin, and ellagic acid reduced the amount of lithocholic acid [112]. However, there was no evidence of lower conversion of primary to secondary BAs when red wine tannins were added to rat diets [113], or when healthy humans received pomegranate juice over a period of four weeks [85]. Regarding sterols, it seems that dietary phenolic compounds could also influence the conversion of cholesterol into their respective microbial derivatives, especially coprostanol. Compared with a control diet, rats that received tea polyphenols and gallotannins increased their excretion of fecal cholesterol in detriment to the excretion of coprostanol [110]. Similarly, pomegranate juice intake reduces the conversion rate of cholesterol to coprostanol in healthy adults [85]. On the other hand, a large proportion of coprostanol was observed in rats after the intake of apple, grape, and red beet juices [111]. These results suggest that the conversion of primary BAs to 
secondary BAs and cholesterol to coprostanol is hampered by phenolic-rich diets, but more data regarding the role of phenolic compounds on colonic sterol gut conversion in humans is needed.

The negative implications of the secondary metabolites of sterols in the colon may be mitigated by phenolic luminal compounds. The inclusion of chlorogenic acid in the diet of mice reduced the deoxycholic acid's tumor-promoting effects [109]. In this line, protection against the cytotoxicity effects of deoxycholic acid in Caco-2 cells was observed when cells were incubated with proanthocyanidins [114]. In another in vitro study in which deoxycholic acid was incubated with HCT-116 cells, antigenotoxic and aticytotoxic activity was observed for quercetin, resveratrol, and rottlerin [115]. In the same study, genistein, curcumin, and epicallocatechin gallate showed no effect.

High fat diets have also been associated with the increase of the activity of $\beta$-glucuronidase [106]. Many toxic metabolites are neutralized by glucuronide conjugation and it is the form that promotes their excretion from the body. Thus, deglucuronidation not only promotes the retention of toxic metabolites in the body but also increases gut environment toxicity. It has been also proposed that phenolic compounds could interfere with the activity of $\beta$-glucuronidase, but the results are inconsistent. A decrease in the activity of $\beta$-glucuronidase has been observed in rats after diet supplementation with grape extract [92] and ellagitannins in combination with cellulose [94]. On the contrary, higher activity of $\beta$-glucuronidase was noticed in rats after the addition of blackcurrant polyphenols to the diet [93].

To date, the effects of phenolic compounds on the microbial transformation of colonic sterols have not been explored in depth and there is no information about the role of phenolic microbial metabolites. Regarding the changes in fecal sterol composition through the effect of phenolic-rich products, the data available in the literature is too scarce to enable solid conclusions to be drawn. Sterols are a non-invasive and easy parameter to be analyzed in fecal samples with a relatively high sensitivity to change when the characteristics of the diet also change [106]. This converts them into good candidates for reflecting alterations in the gut lumen after dietary interventions, particularly those containing high fat.

\subsection{Products of Non-Absorbed Protein Metabolism}

Every day, variable amounts of nitrogenous compounds of dietary and endogenous origins reach the colon. Protein-rich diets, particularly those containing red meat, increase gut toxicity as a consequence of an excessive production of microbial products derived from protein fermentation [64,65,116,117]. These substances, also called putrefactive compounds, include ammonia, fecal phenolic (phenol and $p$-cresol) and indolic compounds (indol and skatole), sulphur compounds (hydrogen sulphide, methyl mercaptan, and dimethyl sulphide), branched chain fatty acids, and polyamides (putrescine, agmatine, cadaverine, tyramine, and histamine). Intestinal ammonia is generated through the microbial deamination of urea and amino acids [65,118]. Phenolic and indolic compounds are mainly derived from the microbial catabolism of aromatic amino acids (phenylalanine, tyrosine, and tryptophan) [65]. Sulphate-reducing bacteria produce sulphur metabolites as a product of anaerobic respiration during the catabolism of cysteine and methionine [119]. Polyamides, besides being present in food, are also released in the gut by the bacterial metabolism [120]. 
Elevated undigested luminal proteins could alter the intestinal ecology, stimulating the activity of nitrogen-degrading bacteria, which are considered to have detrimental effects in gut homeostasis [121,122]. In parallel, microbial enzymatic expression and activity increase proportionally with the amount of substrate [122], thus promoting the accumulation of putrefactive compounds which are associated with gut integrity and function loss due to their potential cytotoxicity, genotoxicity, and carcinogenic activity, increasing the risk of inflammatory bowel disease (IBD) and the development of colorectal cancer $[66,74,116,123]$.

The modification of dietary patterns, such as including dietary carbohydrates [89,116,117], and restricting energy intake [124], has shown an inverse relationship with the concentration of these putrefactive fecal compounds. However, there is insufficient information regarding the effects of phenolic compounds in this aspect. Among the limited number of studies focused on researching the relation between protein fermentation and phenolic compounds, the most numerous are those on polymeric and monomeric flavan-3-ols. In the case of animal studies, a tea phenol-enriched diet reduced the $\mathrm{pH}$ and concentration of ammonia, phenol, $p$-cresol, and skatole in pig feces after two weeks of treatment [81]. Satisfactory results were also observed in humans after supplementation with grape seed extract [125] and flavan-3-ols [126]. Both products reduced fecal $\mathrm{pH}$ and hampered the production of sulphur compounds. A trend toward a decrease in phenol, $p$-cresol, 4-ethylphenol, indole, and skatole in feces was observed after consumption of grape seed extract, but these compounds were not studied after flavan-3-ol supplementation. In the case of ammonia, its fecal amounts were significantly reduced in the flavan-3-ol group and tended to decrease after grape seed extract intake. More consistent results, observed after the intervention with flavan-3-ols, may be related to the duration of the study, this being two and six weeks for grape seed extract and flavan-3-ols treatments, respectively. On the contrary, no changes in the production of ammonia and sulphides were noticed after continuous feeding of soygerm powder in a SHIME system [61].

A reduction of the $\mathrm{pH}$ of the feces observed after phenolic intervention could partly explain the decrease in the concentration of putrefactive substances, since microbial proteases are more active at neutral or slightly alkaline $\mathrm{pH}$ [127]. However, this is not a common effect. In some cases, phenolic compounds increase [92] or have no effects [80,83] on lumen $\mathrm{pH}$. In addition, a reduction in fecal putrefactive compounds does not always respond to a reduction in the $\mathrm{pH}[80,92]$. The differences may depend on the predominant class of bacteria and the availability preference of the substrate type. For example, Escherichia coli and Proteus mirabilis produced ammonia from amino acids even at low $\mathrm{pH}[118]$.

Phenolic compounds and their microbial metabolites possibly collaborate with additional factors to reduce the accumulation of protein fermentation products. A reduction of protein conversion, beyond the decrease in $\mathrm{pH}$ in the gut lumen, may also be associated with the capacity of phenolic compounds to inhibit proteolytic bacteria and interfere with their enzymatic activity. Gram-negative Proteobacteria (especially E. coli), Bacillus spp., and Gram-positive Clostridia [118,128] have been implicated in gut proteolysis and their abundance in IBDs is probably associated with the typical morphological and physiological alterations $[129,130]$. Beside the capacity of phenolic compounds to inhibit the growth of several members of the proteolytic bacteria, it has been observed that phenols also possess the capacity to attenuate the expression of genes involved in the secretion of proteases, inhibiting their 
synthesis and their activity [125,131-133]. The degree to which the enzymatic activity is blocked depends on the phenolic concentration, its molecular structure, and its functional groups [133].

Abnormal concentrations of proteinases and putrefactive compounds in the lumen alter the permeability of the gut epithelium, promoting the movement of toxic substances across the intestinal barrier, which leads to a tendency to activate inflammatory mediators $[129,134]$. One of the reported mechanisms by which phenolic compounds improve barrier integrity is by increasing the expression of tight junction proteins [84,134-137]. This may mean that although some phenolic compounds are unable to reduce protein fermentation, they offer an alternative way of protecting colon integrity, enhancing the host tolerance to susceptible molecules present in the internal environment.

Large intestine digestion is a dynamic system in which entrapped compounds in the digesta pass through the ascending, transversal, and descending colon before being excreted by feces. Local microbiota is active throughout the lumen, metabolizing non-absorbed products in the function of substrate preference and availability, and is also conditioned by the physiological conditions of the gut ( $\mathrm{pH}$, redox potential, transit time) [138]. The pattern of the native phenolic compounds present in food change between the colonic segments due to the microbial fermentation, resulting in the accumulation of different metabolites with different activity than their precursors. As major proteolytic activity is found in the terminal colon $[64,123]$, it would be interesting to study the interaction of microbial proteolysis-phenolic metabolites in this part of the gut. In addition, we have found only one paper related to the study of the modification of fecal nitrogen compounds after the addition of phenolic-rich products in a high red meat diet in humans [139]. However, to have a better understanding of the role of dietary phenolic compounds in protein fermentation at gut level, more human and animal studies are needed.

\section{Impact of Phenolic Compounds on Intestinal Function}

\subsection{Intestinal Redox Homeostasis}

Oxidative stress is defined as the damage promoted by an imbalance between pro-oxidant agents (free radicals) and endogenous (superoxide dismutases, SOD, catalase glutathione peroxidase, GPx, and glutathione, GSH) and exogenous mechanisms to neutralize their effects. Free radicals are highly reactive molecules capable of reacting with the biological components of cellular structures, such as the structural lipids of cell membranes, proteins, and nucleic acids (DNA damage), compromising good cell function and integrity. Moreover, the alteration of cellular components caused by oxidative stress in many cases precedes the over-expression of pro-inflammatory agents that are responsible for the activation of mechanisms that trigger cell damage [140]. Repeated bouts of inflammation can lead to the occurrence of IBDs, such as ulcerative colitis and Crohn's disease, which are, in turn, risk factors of colon cancer [141].

Pro-oxidant substances are products of normal aerobic metabolism, but their levels increase as a consequence of toxic exposure (smoke, excessive alcohol intake, irradiation, some drugs, food components, toxins) and other particular situations (age, infection, stress). The gastrointestinal tract produces, receives, and retains these types of substances, which implies an elevated risk of alterations in cells and tissue in situations of over-production of free radicals and/or depletion of antioxidant 
reparative mechanisms [142]. Dietary phenolic compounds could act as an antioxidant system in the gastrointestinal lumen and epithelium, reducing the oxidative damage in different ways: safeguarding the activity of endogenous antioxidants, decreasing free radical production, or neutralizing the latter by radical scavenging $[6,7,9,140,143-147]$.

In vitro studies using cell-based methods are considered a good tool for deciphering the mechanisms by which phenolic compounds attenuate oxidative responses. Treatment of intestinal Caco-2 cells with different fractions of cranberry and apple peel phenolic extracts [140,143], as well as red wine extracts [144], effectively mitigated the membrane structure disruption caused by the pro-oxidants Fe/ascorbate and tertra-butyl hydroperoxide, respectively. Direct scavenging activity is the proposal mechanism by which urolithins suppress the generation of free radicals from activated neutrophils [145]. On some occasions, foods also collaborate with the pro-oxidant potential of the gut environment. For example, an overproduction of free radicals and a reduction of GSH activity were evident in Caco-2 cells when they were incubated with acrylamide, but the latter effects were reduced by cocoa phenolic extract, procyanidin dimer B2, and epicatechin [146]. In a similar way, protection against oxidative damage induced by the micotoxin deoxynivaleol in HT-29 cells was also observed when the culture media was enriched with epigallocatechin gallate [147].

The main entrance route for phenolic compounds is the gastrointestinal tract, where they reach a greater lumen concentration and remain longer compared with those observed in blood circulation [22,62]. Also, the extensive phase II metabolism (glucuronidation, sulphatation, and methylation) that phenolic compounds undergo during absorption and body distribution created doubts concerning their effectiveness as antioxidant agents at the systemic level [13,145]. In the case of colon lumen, the non-absorbed phenolic compounds and their microbial metabolites prevail in native-unconjugated forms [148]. During digestion, the antioxidant capacity of the luminal content can indeed increase compared with the original food, probably due to the release of phenolic compounds from the food matrix promoted by the physiological conditions and action of digestive enzymes [149]. In the large intestine, microorganisms have the ability to release those phenolic compounds intimately entrapped in the food matrix (particularly dietary fiber), which also contribute to the total antioxidant capacity at the gut lumen level $[150,151]$. Moreover, the phenolic compounds released from the digestive bolus can be distributed into intestinal fluids and favor direct contact with the colonic epithelium, mitigating the detrimental effects of free radicals at the cell level.

The antioxidant status in in vivo studies (animals or humans) could be estimated by analyzing the antioxidant capacity of the intestinal content or fecal water by different methods, such as the oxygen radical absorbing capacity (ORAC), the ferric reducing antioxidant power (FRAP), the diphenyl-1picrylhydrazyl (DPPH), the free radical scavenger (TBARS), and the 2,2'-azinobis-(3ethylbenzothiazoline-6-sulphonate) radical cation (ABTS) [6,13,14,19,20,149-151]. The total antioxidant capacity of the intestinal content increased after the ingestion of grape seed extract in rats [151] and limited the production of free radicals in human fecal water after polyphenol-rich chocolate intake [152]. In contrast, some studies have noted no changes in the antioxidant capacity in colon tissue after the intake of phenolic-rich products [10]. The difference in the bioactivity between microbial phenolic metabolites and their parent compounds together with the progressive decrease of the lumen phenolic concentration due to the active colonic absorption could be an explanation for the unobserved antioxidant effects. However, it should be stressed that lack of effect in the total 
antioxidant capacity does not necessarily encompass the loss of other biological properties [10] (see next Section 4.2.).

\subsection{Intestinal Inflammation}

Inflammation is a biological phenomenon associated with the innate body immune response in the first line of defense to combat threats which could compromise human health. Episodes of controlled inflammation are indispensable for suppressing chemical, biological, and physical injuries. However, in some cases, due to diverse genetic and environmental causes, uncontrolled inflammation responses predispose individuals to the development of chronic diseases. In the case of the gastrointestinal tract, IBDs are characterized by an unknown etymology of recurrent episodes of inflammation causing a progressive loss of cellular function and tissue degeneration that could develop into colon cancer $[141,153]$.

The chronification of inflammation involves complex and interconnected mechanisms of molecular mediators, whose continued feedback promotes the perpetuation of inflammation. The over-production of free radicals (reactive oxygen and nitrogen species) and cytokines (TNF- $\alpha$, IL-6, IL-8, PGE2, among the most studied), the up-regulation of nuclear factor kappa-B (Nf- $\kappa \mathrm{B})$ and monophosphate-activated protein kinase (MAPK), and the recruitment, activation, and adhesion of leukocytes have been identified as occurring in IBDs $[7,8]$. Phenolic compounds seem to inhibit or attenuate the intensity of the inflammatory response by modulating the cellular inflammatory mediators and/or neutralizing free radicals (see Section 4.1 above).

In vitro cultures of intestinal cells are particularly valuable models for identifying the target molecules involved in chronic inflammation which may be susceptible to regulation by phenolic compounds. The results obtained from these studies could be interesting for the design of new anti-inflammatory drugs capable of avoiding the adverse side effects observed for some drugs used to treat IBDs [154]. The possible mechanisms by which phenolic compounds could mitigate or suppress inflammatory responses in vivo can be predicted by cells activated by different types of inflammation promoters. The down-regulation of NF- $\kappa \mathrm{B}$ is considered a therapeutic target in IBDs, since it prevents the activation of pro-inflammatory mediators, such as COX-2 and iNOS, which, in turn, are responsible for the production of cytokines and free radicals. Individual compounds, such as epigallocatechin gallate [147] and anthocyanins [155], and also phenolic mixtures obtained from apple peels [143], cocoa [8], cranberries [140], and red wine [156], have shown an in vitro ability to inhibit the activation of NF-kB pathways. In the latter studies, the inhibition of NF- $\mathrm{B}$ activity was also associated with the down-expression of COX-2 [8,143,147,156] and iNOS [8,140,156]. Resveratrol [157] and cocoa phenolic compounds [146] were also effective as regulators of other signaling pathways such as JAK-STAT and MAPK, respectively. On the contrary, chlorogenic-rich fractions obtained from lyophilized blueberries did not show clear evidence as inhibitor agent of NF- $\kappa$ B [155].

Although less common compared with the data available regarding the anti-inflammatory potential of phenolic compounds, it seems that their microbial metabolites also possess similar properties. The down-regulation of COX-2 in human adenoma cells was observed after the incubation of (3',4'-dihydroxyphenyl)acetic and 3-(3',4'-dihydroxyphenyl)propionic acids [158], common phenolic 
catabolites detected in feces. Reclusion and adhesion of leukocytes play an important role in the development of inflammation since they also secrete pro-inflammatory cytokines. The secretion of cytokines TNF- $\alpha$ and IL- 6 was effectively inhibited by urolithins after activation of THP-1 human monocyte cells [159]. Urolithins were also effective as anti-inflammatory mediators in activated neutrophils, inhibiting cytokines and the secretion of the proteinases necessary for adhesion [145]. Fibroblasts are a type of cell that synthesizes the extracellular matrix and collagen, responsible for tissue cicatrization. Their activation, mediated by cytokines, monocytes, and free radicals, also involves the secretion of pro-inflammatory agents. In addition, the exacerbated extracellular production of collagen could lead to intestinal stenosis (bowel obstruction) [160]. Urolithins, especially urolithin A, were also effective at inhibiting the activation, migration, and adhesion of colon fibroblasts with a concomitant reduction of several secretory pro-inflammatory mediators. These effects were even more evident than those shown by their precursor, ellagic acid [161].

Chemically induced IBD in animals is a revolutionary approach to studying the effect of phenolic compounds on inflammation under in vivo conditions [153]. The improvement in physical indicators (less body weight loss) and hematological and histological probes, together with a reduction in the expression of pro-inflammatory mediators in combination with phenolic treatment, constitute evidence of the protection given by phenolic compounds against intestinal epithelium damage and the attenuation of the inflammatory response. Individual phenolic compounds [7,10,134,136,162], and phenolic extracts $[7,8,163,164]$ have shown positive effects in the control of IBDs in animals, even to the same extent as sulphosalizine, a common medicine used in IBDs, but with fewer collateral effects [7]. Contrary to the later results, the administration of resveratrol to rats for six weeks induces the expression of inflammatory parameters [84]. Data from animal studies support the anti-inflammatory effect of phenolic compounds observed in in vitro experiments, but the role of phenolic metabolites in this aspect is still unclear, due to the lack of information about the phenolic disposition in tissues and feces in most of the later studies.

Dietary intervention in human studies including IBD patients is basically conducted to observe the remission of clinical symptoms or the diminution in the number or intensity of relapses [165-167]. Despite phenolic compounds have been extensively studied in animal models of IBDs, data regarding human trials is currently limited to one study in which the administration of epigallocatechin gallate to patients with ulcerative colitis over 56 days showed a satisfactory degree of remission of symptoms with minimal side effects [166]. Due to the ethical issues mentioned above, human studies to investigate the in situ effects promoted by phenolic compounds are not always possible. Nevertheless, there are promising fecal inflammatory markers that could potentially be used in human studies with minimal invasiveness.

Calprotectin and lactoferrin are binding proteins found in the cytoplasm of neutrophils, monocytes, and macrophages. Fecal calprotectin could be a good tool for the molecular screening of active intestinal inflammation, because its levels are substantially elevated in IBDs and colorectal cancer [167-169]. Furthermore, the amounts of calprotectin in feces have been shown to correlate well with the histological lesions caused by continuous inflammation in IBDs $[167,168,170]$ and the tumor stage in colorectal cancer [169], indeed with better results than other common plasma biomarkers, such as the case of C-reactive protein [167,170]. A reduction in the fecal excretion of calprotectin was observed after surgical intervention [169] and oral probiotic administration [171]. Rats fed with a high fat 
diet in combination with grape seed extract showed lower levels of fecal calprotectin than the control animals [172].

Although collecting feces is easy and non-invasive, human studies concerning fecal inflammation biomarkers are scarce. To our knowledge, there is only one study involving IBDs patients and phenolic treatment. In this work, anthocyanin-rich bilberries were administrated to ulcerative colitis patients for nine weeks and the results at the end of the study showed a decrease in the fecal levels of calprotectin, which increased again when the bilberry treatment was stopped [173]. These results not only show the palliative effects of some phenolic compounds in IBDs, but also the inclusion of calprotectin as an index of inflammation response after dietary treatment. Exploring the association between phenolic compounds and intestinal inflammation through dietary treatment is a promising future approach to be considered.

\section{Phenolic Compounds and Gut Microbiota Modulation}

The gastrointestinal tract, especially the large intestine, houses the most abundant and complex microbiota in humans. The gut population, composed of approximately $10^{10}-10^{12}$ cells per gram of intestinal content, participates in several metabolic functions that the host cannot fulfill by itself [174-176]. These metabolic functions and their derivative end products depend on the quantitative and qualitative features of the gut inhabitants. A harmonious balance in the composition of the gut microbiota has been associated with maintaining health and a higher life expectancy accompanied by a satisfactory quality of life. An imbalance in the microbial population is known as dysbiosis. Dysbiosis has been associated with intestinal and non-intestinal metabolic disorders, which confirms the association between the microbiota and non-digestive functions [176].

Although full characterization of the microbiota in homeostasis and disease still remains to be completed, some trends in its profile could be helpful for discriminating between the two states. A lower diversity of bacteria, change in bacteria functionality, and a decrease in the beneficial inhabitants together with a major abundance of detrimental opportunistic bacteria seem to be involved in metabolic alterations such as obesity and type-2 diabetes, and diseases of the gut such as IBDs and colorectal cancer $[9,10,73,100,177,178]$. An alteration of the microbial equilibrium undermines bowel functionality, alters host immunity, and increases susceptibility to pathogen colonization.

The mechanisms by which the phenolic compounds modulate the gut microbiota still remain to be deciphered, but may involve direct and indirect interactions. Phenolic compounds could directly stimulate or inhibit bacterial growth. Inhibition is closely related to previously reported antimicrobial properties of phenolic compounds and stimulation presumably associated with the capacity of the bacteria to metabolize them [131,179]. The antimicrobial activity of phytochemicals has been extensively studied because is thought that it is the main cause by which phenolic compounds can modify the characteristics of the gut structure population. On the basis of several works [131], it could be said that phenolic compounds possess a selectively bacteriostatic or bactericide effect, inhibiting the growth of a wide range of potential pathogenic bacteria slightly affecting or even promoting the beneficial microbial population. However, it is important to consider the concentration and characteristics of the molecule (type of phenolic compound and if it is presented in conjugated or free form) because these factors seem to govern the modulation of the desired effects. Indirect implications 
include complex issues where numerous microbial-host and microbial-microbial interrelationships take place. Phenolic metabolites could affect the growth of other bacterial groups and, in turn, the over-growth of some bacterial groups could condition the development of others $[76,179,180]$.

The prevalence of determinate microbiota members is preferred to others due to the efficacy they have shown in ameliorating the gut ecosystem with positive effects at the local and systemic levels. For this reason, most studies have focused on the effects of polyphenols on Bifidobacterium and Lactobacillus, but there is increasing interest in other emergent bacteria that could be relevant for the health of the host [9,101]. Under in vitro conditions, anthocyanins [38], phenolic compounds bound to the insoluble cocoa fraction [150], pomegranate extract [45], and soygerm powder [61] have stimulated the growth of Lactobacillus and Bifidobacterium. Pomegranate extract and apple pomace juice were also capable of increasing Bifidobacterium levels in rodents [111,163]. The Lactobacillus count was increased after the administration of apple and red beet pomace juice instead of water in rats [110]. Grape seed extract rich in proanthocyanins was able to increase the population of Bifidobacterium in healthy adults [125]. Although the phenolic compounds contained in a cocoa powder did not prevent the age-induced reduction of Lactobacillus and Bifidobacterium in rats after six weeks, they reduced the growth of Bacteroidetes, Staphylococcus, and Clostridium [181]. The latter prebiotic effects could also have been related to the possible presence of fiber in the phenolic sources. Dietary fiber is known to influence the microbiota profile, especially through the increase in probiotic microorganisms [182]. However, other sources deprived of dietary fiber, as in the case of incubation of individual compounds [76], green tea phenolic compounds in pigs [81], fiber-free juice [101], resveratrol administrated to rats [10], isoflavone supplementation in women [183], and cocoa-isolated phenolic compounds in healthy adults [184], have also shown positive effects to favor the increase in the number of Bifidobacterium [10,76,183,184], Lactobacillus [10,101,184], and butyrate-producing bacteria in parallel with the modulation of growth of bacteria associated with gut dysbiosis [10,76,184].

Diet is probably the most easily manipulable factor to modulate the composition of the gut microbiota, and the latter results show that phenolic compounds per se are presumably able to interact with gut inhabitants and produce changes, most of them toward a healthier profile. However, in a recent study by our group [85], no changes were observed in the microbiota composition of healthy adults after four weeks of sustained intake of pomegranate juice with a high phenolic content. Therefore, the production of the microbial phenolic metabolites catechol and phenylpropionic acid was correlated with the presence of a higher percentage of some bacterial groups, supporting the existence of phenolic compound-bacteria interrelationships. Despite this fact, the moderate consumption of red wine by healthy volunteers over a period of 20 days increased the number of Firmicutes, Bacteroidetes, Bifidubacterium, and Prevotella [185]. The Bifidobacterium population also increased in the feces of healthy humans after six weeks of sustained intake of blueberry drink [186], basically promoted by the higher amounts of some species [187].

Recent studies have proposed the use of phenolic-rich sources as a therapeutic strategy to prevent and, in some cases, reverse the dysbiosis associated with different pathologies. Obese rodents or animals fed with obesogenic diets are common tools for studying the role of the microbiota in the metabolic syndrome. The metabolic syndrome includes a series of alterations, such as obesity, dyslipidaemia, glycosemia, and loss of insulin sensitivity. Thus, the attenuation of one of the latter factors could be considered as a protective factor of type 2 diabetes and cardiovascular diseases. A 
high Firmicutes/Bacteroidetes ratio has been considered an index of obesity and its depletion a marker of successful treatment. However, the results in this context are ambiguous. The reduction in the incidence of obesity reflected by lesser weight gain and fat accumulation was observed after treatment with quercetin plus resveratrol [84], coffee [188], fiber-free plum [101] and cranberry extract [9], but no significant differences were observed after pomegranate peel extract intake by obese mice [163]. The majority of the latter phenolic treatments showed a modification of the microbial population with respect to their control counterparts, but it was not always encompassed by a decrease in the Firmicutes/Bacteroidetes ratio, suggesting the involvement of other bacteria or non-microbiota mediated effects. Nevertheless, a positive correlation between body mass index and the Firmicutes/Bacteroidetes ratio is still questionable because this parameter has not always been described in obesity $[99,101,189]$. The prevention of obesity is a target factor to reduce the incidence of type 2 diabetes, which could be reinforced by the additional effects provided by dietary phenolic compounds. For example, the administration of cranberry extract to mice for nine weeks increased the relative abundance of Akkermansia ssp., which has been linked with the enhancement of diabetic protective effects such as an increase in insulin sensitivity and better inflammatory parameters [9].

IBDs have also been associated with a microbial status in which Enterobacteriacea, especially E. coli, are more abundant than in the control subjects. The characterization of E. coli has revealed that the virulent types were more abundant in biopsy specimens in IBDs patients compared with healthy individuals, basically due to the expression of adhesines which facilitate the adherence of the bacteria to the epithelium surface [190]. The administration of resveratrol to IBD-induced rats for 25 days prevented the over-growth of Enterobacteriaceae, particularly E. coli [10]. Some studies confer the phenolic compounds with the in vitro properties of inhibiting the cellular adhesion of harmful bacteria with a minimal repercussion on the beneficial members of the gut microbiota [191].

All seem to indicate that the gut community responds positively to dietary phenolic compounds where the abundance of determinate bacteria could contribute to host health maintenance. After phenolic intervention, microbiota changes may by expressed on different taxonomic levels. Lack of difference at the phylum level does not necessarily indicate a lack of probiotic effects; sometimes the changes are evident at class, family, genus, or species levels [84].

Microbiota profile and, thus, microbiota modulation after diet intervention can be studied by different techniques, such as fluorescent in situ hybridization (FISH), quantitative PCR-based methods (qPCR) [76,101], and high-throughput sequencing; these are among the most widely used. The analyses by FISH and qPCR are based on identifying and quantifying specific bacterial groups, defined as oligonucleotide probes. For this, in most of the assays, the selected probes depend on the specific bacteria of interest in the study and probably some important microbial changes may be omitted if the probe is not considered in the study. Other, more sophisticated techniques are based on the amplification and pyrosequencing of bacterial $16 \mathrm{~S}$ ribosomal RNA and allow overall population bacterial genoma identification (metagenome) [101,177]. A more in-depth and detailed characterization of the whole microbiome provides information regarding changes not only in the phylum, class, family, and genus but also specific alterations observed at the species level. In summary, the phenolic compounds that may hamper the microbial imbalance in situations of risk of disease or those that may prevent dysbiosis by reinforcing beneficial bacteria could be included in therapeutic strategies to restore or maintain a healthy profile of the gut microbiota. It remains to be 
elucidated whether dysbiosis is a cause or consequence of several human pathologies associated with the microbiota.

\section{Conclusions and Future Perspectives}

The large intestine is a sophisticated and complex ecosystem where interrelationships among the host, microbiota, and its metabolic products (dietary and endogenous products) play a key role in local and systemic health. The inter-dependence between the latter factors is so tight that the alteration of one of them can govern the behavior of the others, altering the gut homeostasis and, thus, making the appearance of disease more likely.

Dietary phenolic compounds reach the colon in variable amounts, enriching the gut lumen of the related metabolites derived from the local microbiota metabolism. Lumen phenolics (parent compounds and their metabolites) can actively take part in the innumerable interactions that occur in the large intestine. Depending on their nature, diet phenolic compounds apparently enhance the generation of beneficial metabolic products and/or hamper the production and effects of detrimental luminal compounds, protecting and prolonging gut homeostasis. The mechanisms by which phenolic compounds can interact with the gut metabolism may involve interference with enzymatic expression and activity, changes in the characteristics of the gut environment, changes in the modulation of the signaling pathways responsible for pro-oxidant, inflammatory, and carcinogenic effects, enhancement in the protection of the intestinal epithelium from the negative effects of toxic substances, and/or the modulation of the microbial population.

There are still several aspects that require emphasizing. Phenolic compounds undergo active colonic microbial transformations, generating intermediate and final related metabolites that could be present in the digesta in higher concentrations than their precursors, without necessarily sharing the same bioactivity. In this context, studies focused on analyzing the parent compounds in food probably fail to estimate the phenomena occurring in the colon correctly. It would be important to identify whether the health benefits are associated with the parent compounds or their respective microbial derivatives in order to design the correct dietetic or technological strategies to obtain major and better results from phenolic dietary therapies.

Phenolic compounds represent a wide group including several sub-groups that, although they have similar characteristics, are not completely equal. Differences in chemical structure, number of functional groups, and the combination of different moieties probably lead to these molecules having different functions. Despite the information obtained and published over recent years, the effects and mechanisms by which phenolic compounds promote changes in the human gut ecosystem are not yet completely elucidated. A solution could be partly promoted by the combination of metabolomic (characterization of metabolite changes in the gut environment after dietary intervention with phenolic compounds or phenol-rich foods), microbiome (gut bacteria collective genome), and metagenomic (for the identification of down- or over-expressed proteins involved in microbial activities, whose classification thus depends on the function) disciplines to describe a more complete scenario promoted by phenolic compounds.

Given all of the above, the design and performance of more research, especially focused on human trials, is encouraged to confirm the efficacy of phenolic compounds at the gut level. The results 
provided by previous and future studies could be useful to the design of dietary recommendations not only to suppress or reduce symptoms in disease but also to provide the healthy population with simple tools to promote the maintenance of health.

\section{Acknowledgments}

This work was supported by the Spanish Ministry of Economy and Competitiveness (Grant AGL2012-40144-C03-03 and Grant SAF2012-31187). JIM was supported by a fellowship from the Generalitat de Catalunya.

\section{Conflicts of Interest}

All authors read and approved the final manuscript and declared that no conflicting interests existed.

\section{References}

1. Acosta-Estrada, B.A.; Gutiérrez-Uribe, J.A.; Serna-Saldívar, S.O. Bound phenolics in foods, a review. Food Chem. 2014, 152, 46-55.

2. Mosele, J.I.; Macià, A.; Romero, M.P.; Motilva, M.J.; Rubió, L. Application of in vitro gastrointestinal digestion and colonic fermentation models to pomegranate products (juice, pulp and peel extract) to study the stability and catabolism of phenolic compounds. J. Funct. Foods 2015, 14, 529-540.

3. Tomás-Barberán, F.A.; García-Villalba, R.; González-Sarrías, A.; Selma, M.V.; Espín, J.C. Ellagic acid metabolism by human gut microbiota: Consistent observation of three urolithin phenotypes in intervention trials, independent of food source, age, and health status. J. Agric. Food Chem. 2014, 62, 6535-6538.

4. González-Barrio, R.; Borges, G.; Mullen, W.; Crozier, A. Bioavailability of anthocyanins and ellagitannins following consumption of raspberries by healthy humans and subjects with an ileostomy. J. Agric. Food Chem. 2010, 58, 3933-3939.

5. Cummings, J.H.; Wiggins H.S.; Jenkins, D.J.A.; Houston, H.; Jivraj, T.; Drasar, B.S.; Hill, M.J. Influence of diets high and low in animal fat on bowel habit, gastrointestinal transit time, fecal microflora, bile acid, and fat excretion. J. Clin. Investig. 1978, 61, 953-963.

6. Soobrattee, M.A.; Neergheen, V.S.; Luximon-Ramma, A.; Aruoma, O.I.; Bahorun, T. Phenolics as potential antioxidant therapeutic agents: Mechanism and actions. Mutat. Res. Fundam. Mol. Mech. Mutagen. 2005, 579, 200-213.

7. Oz, H.S.; Chen, T.; de Villiers, W.J.S. Green tea polyphenols and sulfasalazine have parallel anti-inflammatory properties in colitis models. Front. Immunol. 2013, 4, 132.

8. Rodríguez-Ramiro, I.; Ramos, S.; López-Oliva, E.; Agis-Torres, A.; Bravo, L.; Goya1 L.; Martín, M.A. Cocoa polyphenols prevent inflammation in the colon of azoxymethane-treated rats and in TNF-a-stimulated Caco-2 cells. Br. J. Nutr. 2013, 110, 206-215. 
9. Anhê, F.F.; Roy, D.; Pilon, G.; Dudonné, S.; Matamoros, S.; Varin, T.V.; Garofalo, C.; Moine, Q.; Desjardins, Y.; Levy, E.; et al. A polyphenol-rich cranberry extract protects from diet-induced obesity, insulin resistance and intestinal inflammation in association with increased Akkermansia spp. population in the gut microbiota of mice. Gut 2015, 64, 872-883.

10. Larrosa, M.; Yañéz-Gascón, M.J.; Selma, M.V.; González-Sarriás, A.; Toti, S.; Cerón, J.J.; Tomás-Barberán, F.; Dolara, P.; Espín, J.C. Effect of a low dose of dietary resveratrol on colon microbiota, inflammation and tissue damage in a DSS-induced colitis rat model. J. Agric. Food Chem. 2009, 57, 2211-2220.

11. Roowi, S.; Stalmach, A.; Mullen, W.; Lean, M.E.J.; Edwards, C.A.; Crozier, A. Green tea flavan-3-ols: Colonic degradation and urinary excretion of catabolites by humans. J. Agric. Food Chem. 2010, 58, 1296-1304.

12. Barroso, E.; van de Wiele, T.; Jiménez-Girón, A.; Muñoz-González, I.; Martín-Alvarez, P.J.; Moreno-Arribas, M.V.; Bartolomé, B.; Peláez, C.; Martínez-Cuesta, M.C.; Requena, T. Lactobacillus plantarum IFPL935 impacts colonic metabolism in a simulator of the human gut microbiota during feeding with red wine polyphenols. Appl. Microbiol. Biotechnol. 2014, 98, 6805-6815.

13. Monagas, M.; Urpi-Sarda, M.; Sánchez-Patán, F.; Llorach, R.; Garrido, I.; Gómez-Cordovés, C.; Andres-Lacueva, C.; Bartolomé, B. Insights into the metabolism and microbial biotransformation of dietary flavan-3-ols and the bioactivity of their metabolites. Food Funct. 2010, 1, 233-253.

14. Sánchez-Patán, F.; Cueva, C.; Monagas, M.; Walton, G.E.; Gibson, G.R.; Martín-Álvarez, P.J.; Victoria Moreno-Arribas, M.; Bartolomé, B. Gut microbial catabolism of grape seed flavan-3-ols by human faecal microbiota. Targeted analysis of precursor compounds, intermediate metabolites and end-products. Food Chem. 2012, 131, 337-347.

15. Cueva, C.; Sánchez-Patán, F.; Monagas, M.; Walton, G.E.; Gibson, G.R.; Martín-Álvarez, P.J.; Bartolomé, B.; Moreno-Arribas, M.V. In vitro fermentation of grape seed flavan-3-ol fractions by human faecal microbiota: Changes in microbial groups and phenolic metabolites. FEMS Microb. Ecol. 2013, 83, 792-805.

16. Van Duynhoven, J.; van der Hooft, J.J.J.; van Dorsten, F.A.; Peters, S.; Foltz, M.; Gomez-Roldan, V.; Vervoort, J.; de Vos, R.C.H.; Jacobs, D.M. Rapid and sustained systemic circulation of conjugated gut microbial catabolites after single-dose black tea extract consumption. J. Proteome Res. 2014, 13, 2668-2678.

17. Van Dorsten, F.A.; Peters, S.; Gross, G.; Gomez-Roldan, V.; Klinkenberg, M.; de Vos, R.C.; Vaughan, E.E.; van Duynhoven, J.P.; Possemiers, S.; van de Wiele, T.; et al. Gut microbial metabolism of polyphenols from black tea and red wine/grape juice is source-specific and colon-region dependent. J. Agric. Food Chem. 2012, 60, 11331-11342.

18. Margalef, M.; Pons, Z.; Bravo, F.I.; Muguerza, B.; Arola-Arnal, A. Plasma kinetics and microbial biotransformation of grape seed flavanols in rats. J. Funct. Foods 2015, 12, 478-488.

19. Jaganath, I.B.; Mullen, W.; Lean, M.E.J.; Edwards, C.A.; Crozier, A. In vitro catabolism of rutin by human fecal bacteria and the antioxidant capacity of its catabolites. Free Radic. Biol. Med. 2009, 47, 1180-1189. 
20. Peng, X.; Zhang, Z.; Zhang, N.; Liu, L.; Li, S.; Wei, H. In vitro catabolism of quercetin by human fecal bacteria and the antioxidant capacity of its catabolites. Food Nutr. Res. 2014, $58,23406$.

21. Schneider, H.; Blaut, M. Anaerobic degradation of flavonoids by Eubacterium ramulus. Archives Microb. 2000, 173, 71-75.

22. Mullen, W.; Rouanet, J.-M.; Auger, C.; Teissèdre, P.-L.; Caldwell, S.T.; Hartley, R.C.; Lean, M.E.J.; Edwards, C.A.; Crozier, A. Bioavailability of [2-14C]quercetin-4'-glucoside in rats. J. Agric. Food Chem. 2008, 56, 12127-12137.

23. Rechner, A.R.; Smith, M.A.; Kuhnle, G.; Gibson, G.R.; Debnam, E.S.; Srai, S.K.S.; Moore, K.P.; Rice-Evans, C.A. Colonic metabolism of dietary polyphenols: Influence of structure on microbial fermentation products. Free Radic. Biol. Med. 2004, 36, 212-225.

24. Lin, Y.; Wu, B.; Li, Z.; Hong, T.; Chen, M.; Tan, Y.; Jiang, J.; Huang, C. Metabolite identification of myricetin in rats using HPLC coupled with ESI-MS. Chromatographia 2012, 75, 655-660.

25. Du, L.-Y.; Zhao, M.; Xu, J.; Qian, D.-W.; Jiang, S.; Shang, E.-X.; Guo, J.-M.; Liu, P.; Su, S.-L.; Duan, J.-A.; et al. Identification of the metabolites of myricitrin produced by human intestinal bacteria in vitro using ultra-performance liquid chromatography/quadrupole time-of-flight mass spectrometry. Expert Opin. Drug Metab. Toxicol. 2014, 10, 921-931.

26. Mosele, J.I.; Martín-Peláez, S.; Macià, A.; Farràs, M.; Valls, R.M.; Catalán, U.; Motilva, M.J. Study of the catabolism of thyme phenols combining in vitro fermentation and human intervention. J. Agric. Food Chem. 2014, 62, 10954-10961.

27. Pereira-Caro, G.; Borges, G.; Ky, I.; Ribas, A.; Calani, L.; Del Rio, D.; Clifford, M.N.; Roberts, S.A.; Crozier, A. In vitro colonic catabolism of orange juice (poly)phenols. Mol. Nutr. Food Res. 2015, 59, 465-475.

28. Miyake, Y.; Yamamoto, K.; Osawa, T. Metabolism of antioxidant in lemon fruit (Citrus limon BURM.f.) by human intestinal bacteria. J. Agric. Food Chem. 1997, 45, 3738-3742.

29. Hanske, L.; Loh, G.; Sczesny, S.; Blaut, M.; Braune, A. The bioavailability of apigenin-7-glucoside is influenced by human intestinal microbiota in rats. J. Nutr. 2009, 139, 1095-1102.

30. Braune, A.; Gütschow, M.; Engst, W.; Blaut, M. Degradation of quercetin and luteolin by Eubacterium ramulus. Appl. Env. Microb. 2001, 67, 5558-5567.

31. Matthies, A.; Blaut, M.; Braune, A. Isolation of a human intestinal bacterium capable of daidzein and genistein conversion. Appl. Env. Microb. 2009, 75, 1740-1744.

32. Matthies, A.; Loh, G.; Blaut, M.; Braune, A. Daidzein and genistein are converted to equol and 5-hydroxy-equol by human intestinal slackia isoflavoniconvertens in gnotobiotic rats. J. Nutr. 2012, 142, 40-46.

33. Coldham, N.G.; Darby, C.; Hows, M.; King, L.J.; Zhang, A.-Q.; Sauer, M.J. Comparative metabolism of genistin by human and rat gut microflora: Detection and identification of the end-products of metabolism. Xenobiotica 2002, 32, 45-62.

34. Walsh, K.R.; Haak, S.J.; Bohn, T.; Tian, Q.; Schwartz, S.J.; Failla, M.L. Isoflavonoid glucosides are deconjugated and absorbed in the small intestine of human subjects with ileostomies. Am. J. Clin. Nutr. 2007, 85, 1050-1056. 
35. Setchell, K.D.R.; Brown, N.M.; Lydeking-Olsen, E. The clinical importance of the metabolite equol-A clue to the effectiveness of soy and its isoflavones. J. Nutr. 2002, 132, 3577-3584.

36. Aura, A.-M.; Martin-Lopez, P.; O’Leary, K.A.; Williamson, G.; Oksman-Caldentey, K.-M.; Poutanen, K.; Santos-Buelga, C. In vitro metabolism of anthocyanins by human gut microflora. Eur. J. Nutr. 2005, 44, 133-142.

37. González-Barrio, R.; Edwards, C.A.; Crozier, A. Colonic catabolism of ellagitannins, ellagic acid, and raspberry anthocyanins: In vivo and in vitro studies. Drug Metab. Disposition 2011, 39, 1680-1688.

38. Hidalgo, M.; Oruna-Concha, M.J.; Kolida, S.; Walton, G.E.; Kallithraka, S.; Spencer, J.P.E.; Gibson, G.R.; de Pascual-Teresa, S. Metabolism of anthocyanins by human gut microflora and their influence on gut bacterial growth. J. Agric. Food Chem. 2012, 60, 3882-3890.

39. Forester, S.C.; Waterhouse, A.L. Identification of cabernet sauvignon anthocyanin gut microflora metabolites. J. Agric. Food Chem. 2008, 56, 9299-9304.

40. Fleschhut, J.; Kratzer, F.; Rechkemmer, G.; Kulling, S.E. Stability and biotransformation of various dietary anthocyanins in vitro. Eur. J. Nutr. 2006, 45, 7-18.

41. Tomás-Barberán, F.; García-Villalba, R.; Quartieri, A.; Raimondi, S.; Amaretti, A.; Leonardi, A.; Rossi, M. In vitro transformation of chlorogenic acid by human gut microbiota. Mol. Nutr. Food Res. 2014, 58, 1122-1131.

42. Ludwig, I.A.; Paz de Peña, M.; Concepción, C.; Alan, C. Catabolism of coffee chlorogenic acids by human colonic microbiota. BioFactors 2013, 39, 623-632.

43. Gonthier, M.-P.; Verny, M.-A.; Besson, C.; Rémésy, C.; Scalbert, A. Chlorogenic acid bioavailability largely depends on its metabolism by the gut microflora in rats. J. Nutr. 2003, 133, 1853-1859.

44. Snelders, J.; Olaerts, H.; Dornez, E.; Van de Wiele, T.; Aura, A.-M.; Vanhaecke, L.; Delcour, J.A.; Courtin, C.M. Structural features and feruloylation modulate the fermentability and evolution of antioxidant properties of arabinoxylanoligosaccharides during in vitro fermentation by human gut derived microbiota. J. Funct. Foods 2014, 10, 1-12.

45. Bialonska, D.; Ramnani, P.; Kasimsetty, S.G.; Muntha, K.R.; Gibson, G.R.; Ferreira, D. The influence of pomegranate by-product and punicalagins on selected groups of human intestinal microbiota. Int. J. Food Microb. 2010, 140, 175-182.

46. Espín, J.C.; González-Barrio, R.; Cerdá, B.; López-Bote, C.; Rey, A.I.; Tomás-Barberán, F.A. Iberian pig as a model to clarify obscure points in the bioavailability and metabolism of ellagitannins in humans. J. Agric. Food Chem. 2007, 55, 10476-10485.

47. García-Villalba, R.; Beltrán, D.; Espín, J.C.; Selma, M.V.; Tomás-Barberán, F.A. Time course production of urolithins from ellagic acid by human gut microbiota. J. Agric. Food Chem. 2013, 61, 8797-8806.

48. Selma, M.V.; Beltrán, D.; García-Villalba, R.; Espín, J.C.; Tomás-Barberán, F.A. Description of urolithin production capacity from ellagic acid of two human intestinal Gordonibacter species. Food Funct. 2014, 5, 1779-1784.

49. Bode, L.M.; Bunzel, D.; Huch, M.; Cho, G.-S.; Ruhland, D.; Bunzel, M.; Bub, A.; Franz, C.M.A.P.; Kulling, S.E. In vivo and in vitro metabolism of trans-resveratrol by human gut microbiota. Am. J. Clin. Nutr. 2013, 97, 295-309. 
50. Rotches-Ribalta, M.; Andres-Lacueva, C.; Estruch, R.; Escribano, E.; Urpi-Sarda, M. Pharmacokinetics of resveratrol metabolic profile in healthy humans after moderate consumption of red wine and grape extract tablets. Pharmacol. Res. 2012, 66, 375-382.

51. Xie, L.-H.; Akao, T.; Hamasaki, K.; Deyama, T.; Hattori, M. Biotransformation of pinoresinol diglucoside to mammalian lignans by human intestinal microflora, and isolation of Enterococcus faecalis strain PDG-1 responsible for the transformation of (+)-pinoresinol to $(+)$-lariciresinol. Chem. Pharm. Bull. 2003, 51, 508-515.

52. Eeckhaut, E.; Struijs, K.; Possemiers, S.; Vincken, J.-P.; de Keukeleire, D.; Verstraete, W. Metabolism of the lignan macromolecule into enterolignans in the gastrointestinal lumen as determined in the simulator of the human intestinal microbial ecosystem. J. Agric. Food Chem. 2008, 56, 4806-4812.

53. Aura, A.M.; Oikarinen, S.; Mutanen, M.; Heinonen, SM.; Adlercreutz, H.C.T.; Virtanen, H.; Poutanen, K.S. Suitability of a batch in vitro fermentation model using human faecal microbiota for prediction of conversion of flaxseed lignans to enterolactone with reference to an in vivo rat model. Eur. J. Nutr. 2006, 45, 45-51.

54. Heinonen, S.; Nurmi, T.; Liukkonen, K.; Poutanen, K.; Wähälä, K.; Deyama, T.; Nishibe, S.; Adlercreutz, H. In vitro metabolism of plant lignans: New precursors of mammalian lignans enterolactone and enterodiol. J. Agric. Food Chem. 2001, 49, 3178-3186.

55. Mosele, J.I.; Martín-Peláez, S.; Macià, A.; Farràs, M.; Valls, R.M.; Catalán, U.; Motilva, M.J. Faecal microbial metabolism of olive oil phenolic compounds: In vitro and in vivo approaches. Mol. Nutr. Food Res. 2014, 58, 1809-1819.

56. Corona, G.; Tzounis, X.; Dessì, M.A.; Deiana, M.; Debnam, E.S.; Visioli, F.; Spencer, J.P.E. The fate of olive oil polyphenols in the gastrointestinal tract: Implications of gastric and colonic microflora-dependent biotransformation. Free Radic. Res. 2006, 40, 647-658.

57. Lin, P.; Qian, W.; Wang, X.; Cao, L.; Li, S.; Qian, T. The biotransformation of oleuropein in rats. Biomed. Chromatogr. 2013, 27, 1162-1167.

58. Pero, R.W. Health consequences of catabolic synthesis of hippuric acid in humans. Curr. Clin. Pharmacol. 2010, 5, 67-73.

59. Lees, H.J.; Swann, J.R.; Wilson, I.D.; Nicholson, J.K.; Holmes, E. Hippurate: The natural history of a mammalian-microbial cometabolite. J. Proteome Res. 2013, 12, 1527-1546.

60. Zheng, X.; Xie, G.; Zhao, A.; Zhao, L.; Yao, C.; Chiu, N.H.L.; Zhou, Z.; Bao, Y.; Jia, W.; Nicholson, J.K.; et al. The footprints of gut microbial-mammalian co-metabolism. J. Proteome Res. 2011, 10, 5512-5522.

61. De Boever, P.; Deplancke, B.; Verstraete, W. Fermentation by gut microbiota cultured in a simulator of the human intestinal microbial ecosystem is improved by supplementing a soygerm powder. J. Nutr. 2000, 130, 2599-2606.

62. Stalmach, A.; Edwards, C.A.; Wightman, J.D.; Crozier, A. Gastrointestinal stability and bioavailability of (poly)phenolic compounds following ingestion of Concord grape juice by humans. Mol. Nutr. Food Res. 2012, 56, 497-509.

63. Verbeke, K.A.; Boobis, A.R.; Chiodini, A.; Edwards, C.A.; Franck, A.; Kleerebezem, M.; Nauta, A.; Raes, J.; van Tol, E.A.F.; Tuohy, K.M. Towards microbial fermentation metabolites as markers for health benefits of prebiotics. Nutr. Res. Rev. 2015, 28, 42-66. 
64. Macfarlane, G.T.; Gibson, G.R.; Beatty, E.; Cummings, J.H. Estimation of short-chain fatty acid production from protein by human intestinal bacteria based on branched-chain fatty acid measurements. FEMS Microb. Ecol. 1992, 10, 81-88.

65. Smith, E.A.; Macfarlane, G.T. Dissimilatory amino acid metabolism in human colonic bacteria. Anaerobe 1997, 3, 327-337.

66. Van Nuenen, M.H.M.C.; Venema, K.; van der Woude, J.C.J.; Kuipers, E.J. The metabolic activity of fecal microbiota from healthy individuals and patients with inflammatory bowel disease. Dig. Dis. Sci. 2004, 49, 485-491.

67. Tiihonen, K.; Ouwehand, A.C.; Rautonen, N. Effect of overweight on gastrointestinal microbiology and immunology: Correlation with blood biomarkers. Br. J. Nutr. 2010, 103, 1070-1078.

68. Wong, J.M.W.; de Souza, R.; Kendall, C.W.C.; Emam, A.; Jenkins, D.J.A. Colonic health: Fermentation and short chain fatty acids. J. Clin. Gastroent. 2006, 40, 235-243.

69. Barcelo, A.; Claustre, J.; Moro, F.; Chayvialle, J.-A.; Cuber, J.-C.; Plaisancié, P. Mucin secretion is modulated by luminal factors in the isolated vascularly perfused rat colon. Gut 2000, 46, 218-224.

70. Clausen, M.R.; Mortensen, P.B. Kinetic studies on the metabolism of short-chain fatty acids and glucose by isolated rat colonocytes. Gastroenterology 1994, 106, 423-432.

71. Waldecker, M.; Kautenburger, T.; Daumann, H.; Busch, C.; Schrenk, D. Inhibition of histone-deacetylase activity by short-chain fatty acids and some polyphenol metabolites formed in the colon. J. Nutr. Biochem. 2008, 19, 587-593.

72. Monleón, D.; Manuel Morales, J.; Barrasa, A.; López, J.A.; Vázquezc, C.; Celda, B. Metabolite profiling of fecal water extracts from human colorectal cancer. NMR Biomed. 2009, 22, 342-348.

73. Balamurugan, R.; Rajendiran, E.; George, S.; Samuel, G.V.; Ramakrishna, B.S. Real-time polymerase chain reaction quantification of specific butyrate-producing bacteria, Desulfovibrio and Enterococcus faecalis in the feces of patients with colorectal cancer. J. Gastroenterol. Hepatol. (Aust.) 2008, 23, 1298-1303.

74. Kanazawa, K.; Konishi, F.; Mitsuoka, T.; Terada, A.; Itoh, K.; Narushima, S.; Kumemura, M.; Kimura, H. Factors influencing the development of sigmoid colon cancer: Bacteriologic and biochemical studies. Cancer 1996, 77, 1701-1706.

75. Jiang, Z.; Fanger, G.R.; Banner, B.F.; Woda, B.A.; Algate, P.; Dresser, K.; Xu, J.; Reed, S.G.; Rock, K.L.; Chu, P.G. A dietary enzyme: $\alpha$-Methylacyl-CoA racemase/P504S is overexpressed in colon carcinoma. Cancer Detect. Prev. 2003, 27, 422-426.

76. Parkar, S.G.; Trower, T.M.; Stevenson, D.E. Fecal microbial metabolism of polyphenols and its effects on human gut microbiota. Anaerobe 2013, 23, 12-19.

77. Zampa, A.; Silvi1, S.; Servili, M.; Montedoro, G.; Orpianesi, C.; Cresci, A. In vitro modulatory effects of colonic microflora by olive oil iridoids. Microb. Ecol. Health Dis. 2006, 18, 147-153.

78. Bazzocco, S.; Mattila, I.; Guyot, S.; Renard, C.M.G.C.; Aura, A.-M. Factors affecting the conversion of apple polyphenols to phenolic acids and fruit matrix to short-chain fatty acids by human faecal microbiota in vitro. Eur. J. Nutr. 2008, 47, 442-452. 
79. Brune, A.; Miambi, E.; Breznak, J.A. Roles of oxygen and the intestinal microflora in the metabolism of lignin-derived phenylpropanoids and other monoaromatic compounds by termites. Appl. Environ. Microbiol. 1995, 61, 2688-2695.

80. Kosmala, M.; Zduńczyk, Z.; Jü̈kiewicz, J.; Jurgoński, A.; Karlińska, E.; Macierzyński, J.; Jańczak, R.; Rój, E. Chemical composition of defatted strawberry and raspberry seeds and the effect of these dietary ingredients on polyphenol metabolites, intestinal function, and selected serum parameters in rats. J. Agric. Food Chem. 2015, 63, 2989-2996.

81. Hara, H.; Orita, N.; Hatano, S.; Ichikawa, H.; Hara, Y.; Matsumoto, N.; Kimura, Y.; Terada, A.; Mitsuoka, T. Effect of tea polyphenols on fecal flora and fecal metabolic products of pigs. J. Vet. Med. Sci. Jpn. Soc. Vet. Sci. 1995, 57, 45-49.

82. Jacobs, D.M.; Deltimple, N.; van Velzen, E.; van Dorsten, F.A.; Bingham, M.; Vaughan, E.E.; van Duynhoven, J. ${ }^{1} \mathrm{H}$ NMR metabolite profiling of feces as a tool to assess the impact of nutrition on the human microbiome. NMR Biomed. 2008, 21, 615-626.

83. Da Silva, J.K.; Cazarin, C.B.B.; Colomeu, T.C.; Batista, T.G.; Meletti, L.M.M.; Paschoal, J.A.R.; Bogusz Júnior, S.; Furlan, M.F.; Reyes, F.G.R.; Augusto, F.; et al. Antioxidant activity of aqueous extract of passion fruit (Passiflora edulis) leaves: In vitro and in vivo study. Food Res. Int. 2013, 53, 882-890.

84. Etxeberria, U.; Ariasc, N.; Boqué, N.; Macarullac, M.T.; Portillo, M.P.; Martíneza, J.A.; Milagro, F.I. Reshaping faecal gut microbiota composition by the intake of trans-resveratrol and quercetin in high-fat sucrose diet-fed rats. J. Nutr. Biochem. 2015, 26, 651-660.

85. Mosele, J.I.; Gosalbes, M.J.; Macià, A.; Rubió, L.; Vázquez-Castellanos, J.F.; Jiménez Hernández, N.; Moya, A.; Latorre, A.; Motilva, M.J. Effect of daily intake of pomegranate juice on fecal microbiota and feces metabolites from healthy volunteers. Mol. Nutr. Food Res. 2015, in press.

86. Koecher, K.J.; Noack, J.A.; Timm, D.A.; Klosterbuer, A.S.; Thomas, W.; Slavin, J.L. Estimation and interpretation of fermentation in the Gut: Coupling results from a $24 \mathrm{~h}$ batch in vitro system with fecal measurements from a human intervention feeding study using fructo-oligosaccharides, inulin, gum acacia, and pea fiber. J. Agric. Food Chem. 2014, 62, 1332-1337.

87. Jakobsdottir, G.; Nilsson, U.; Blanco, N.; Sterner, O.; Nyman, M. Effects of soluble and insoluble fractions from bilberries, black currants, and raspberries on short-chain fatty acid formation, anthocyanin excretion, and cholesterol in rats. J. Agric. Food Chem. 2014, 62, 4359-4368.

88. Juśkiewicz, J.; Milala, J.; Jurgoński, A.; Król, B.; Zduńczyk, Z. Consumption of polyphenol concentrate with dietary fructo oligosaccharides enhances cecal metabolism of quercetin glycosides in rats. Nutrition 2011, 27, 351-357.

89. Aprikian, O.; Duclos, V.; Guyot, S.; Besson, C.; Manach, C.; Bernalier, A.; Morand, C.; Rémésy, C.; Demigné, C. Apple pectin and a polyphenol-rich apple concentrate are more effective together than separately on cecal fermentations and plasma lipids in rats. J. Nutr. 2003, $133,1860-1865$.

90. Wallace, A.J.; Eady, S.L.; Hunter, D.C.; Skinner, M.A.; Huffman, L.; Ansell, J.; Blatchford, P.; Wohlers, M.; Herath, T.D.; Hedderley, D.; et al. No difference in fecal levels of bacteria or short chain fatty acids in humans, when consuming fruit juice beverages containing fruit fiber, fruit polyphenols, and their combination. Nutr. Res. 2015, 35, 23-34. 
91. Kosmala, M.; Kolodziejczyk, K.; Zduńczyk, Z.; Juśkiewicz, J.; Boros, D. Chemical composition of natural and polyphenol-free apple pomace and the effect of this dietary ingredient on intestinal fermentation and serum lipid parameters in rats. J. Agric. Food Chem. 2011, 59, 9177-9185.

92. Zduńczyk, Z.; Juśkiewicz, J.; Estrella, I. Cecal parameters of rats fed diets containing grapefruit polyphenols and inulin as single supplements or in a combination. Nutrition 2006, 22, 898-904.

93. Kosmala, M.; Zduńczyk, Z.; Karlińska, E.; Juśkiewicz, J. The effects of strawberry, blackcurrant, and chokeberry extracts in a grain dietary fiber matrix on intestinal fermentation in rats. Food Res. Int. 2014, 64, 752-761.

94. Fotschki, B.; Milala, J.; Jurgoński, A.; Karlińska, E.; Zduńczyk, Z.; Juśkiewicz, J. Strawberry ellagitannins thwarted the positive effects of dietary fructooligosaccharides in rat cecum. J. Agric. Food Chem. 2014, 62, 5871-5880.

95. Wang, D.; Williams, B.A.; Ferruzzi, M.G.; D’Arcy, B.R. Different concentrations of grape seed extract affect in vitro starch fermentation by porcine small and large intestinal inocula. J. Sci. Food Agric. 2013, 93, 276-283.

96. Kemperman, R.A.; Gross, G.; Mondot, S.; Possemiers, S.; Marzorati, M.; van de Wiele, T.; Doré, J.; Vaughan, E.E. Impact of polyphenols from black tea and red wine/grape juice on a gut model microbiome. Food Res. Int. 2013, 53, 659-669.

97. Aura, A.-M.; Niemi, P.; Mattila, I.; Niemelä, K.; Smeds, A.; Tamminen, T.; Faulds, C.; Buchert, J.; Poutanen, K. Release of small phenolic compounds from Brewer's spent grain and its lignin fractions by human intestinal microbiota in vitro. J. Agric. Food Chem. 2013, 61, 9744-9753.

98. McNeil, N.I. The contribution of the large intestine to energy supplies in man. Am. J. Clin. Nutr. 1984, 39, 338-342.

99. Schwiertz, A.; Taras, D.; Schäfer, K.; Beijer, S.; Bos, N.A.; Donus, C.; Hardt, P.D. Microbiota and SCFA in lean and overweight healthy subjects. Obesity 2009, 18, 190-195.

100. Ley, R.E.; Turnbaugh, P.J.; Klein, S.; Gordon, J.I. Microbial ecology: Human gut microbes associated with obesity. Nature 2006, 444, 1022-1023.

101. Noratto, G.D.; Garcia-Mazcorro, J.F.; Markel, M.; Martino, H.S.; Minamoto, Y.; Steiner, J.M.; Byrne, D.; Suchodolski, J.S.; Mertens-Talcott, S.U. Carbohydrate-free peach (Prunus persica) and plum (Prunus domestica) juice affects fecal microbial ecology in an obese animal model. PLOS ONE 2014, 9, e101723.

102. Macdonald, I.A.; Bokkenheuser, V.D.; Winter, J.; McLernon, A.M.; Mosbach, E.H. Degradation of steroids in the human gut. J. Lipid Res. 1983, 24, 675-700.

103. Hirano, S.; Masuda, N.; Oda, H.; Imamura, T. Transformation of bile acids by mixed microbial cultures from human feces and bile acid transforming activities of isolated bacterial strains. Microb. Immunol. 1981, 25, 271-282.

104. Dewei, R.; Ling, L.; Schwabacher, A.W.; Young, J.W.; Beitz, D.C. Mechanism of cholesterol reduction to coprostanol by Eubacterium coprostanoligenes ATCC 51222. Steroids 1996, 61, 33-40.

105. Montilla, P.; Espejo, I.; Muñoz, M.C.; Bujalance, I.; Muñoz-Castañeda, J.R.; Túnez, I. Effect of red wine on oxidative stress and hypercholesterolemia induced by feeding a high-cholesterol diet in rat. J. Physiol. Biochem. 2004, 60, 259-264. 
106. Reddy, B.S.; Hanson, D.; Mangat, S.; Mathews, L.; Sbaschnig, M.; Sharma, C.; Simi, B. Effect of high-fat, high-beef diet and of mode of cooking of beef in the diet on fecal bacterial enzymes and fecal bile acids and neutral sterols. J. Nutr. 1980, 110, 1880-1887.

107. Kakiyama, G.; Hylemon, P.B.; Zhou, H.; Pandak, W.M.; Heuman, D.M.; Kang, D.J.; Takei, H.; Nittono, H.; Ridlon, J.M.; Fuchs, M.; et al. Colonic inflammation and secondary bile acids in alcoholic cirrhosis. Am. J. Physiol. Gastrointest. Liver Physiol. 2014, 306, G929-G937.

108. Bernstein, H.; Bernstein, C.; Payne, C.M.; Dvorakova, K.; Garewal, H. Bile acids as carcinogens in human gastrointestinal cancers. Mutat. Res. 2005, 589, 47-65.

109. Bernstein, C.; Holubec, H.; Bhattacharyya, A.K.; Huy Nguyen, H.; Payne, C.M.; Zaitlin, B.; Bernstein, H. Carcinogenicity of deoxycholate, a secondary bile acid. Arch. Toxicol. 2011, 85, 863-871.

110. Nakamura, Y.; Kaihara, A.; Yoshii, K.; Tsumura, Y.; Ishimitsu, S.; Tonogai, Y. Effects of the oral administration of green tea polyphenol and tannic acid on serum and hepatic lipid contents and fecal steroid excretion in rats. J. Health Sci. 2001, 47, 107-117.

111. Sembries, S.; Dongowski, G.; Mehrländer, K.; Will, F.; Dietrich, H. Physiological effects of extraction juices from apple, grape, and red beet pomaces in rats. J. Agric. Food Chem. 2006, 54, 10269-10280.

112. Han, Y.; Haraguchi, T.; Iwanaga, S.; Tomotake, H.; Okazaki, Y.; Mineo, S.; Moriyama, A.; Inoue, J.; Kato, N. Consumption of some polyphenols reduces fecal deoxycholic acid and lithocholic acid, the secondary bile acids of risk factors of colon cancer. J. Agric. Food Chem. 2009, 57, 8587-8590.

113. Caderni, G.; Remy, S.; Cheynier, V.; Morozzi, G.; Dolara, P. Effect of complex polyphenols on colon carcinogenesis. Eur. J. Nutr. 1999, 38, 126-132.

114. Erlejman, A.G.; Fraga, C.G.; Oteiza, P.I. Procyanidins protect Caco-2 cells from bile acid- and oxidant-induced damage. Free Radic. Biol. Med. 2006, 41, 1247-1256.

115. Longpre, J.M.; Loo, G. Protection of human colon epithelial cells against deoxycholate by rottlerin. Apoptosis 2008, 13, 1162-1171.

116. Winter, J.; Nyskohus, L.; Young, G.P.; Hu, Y.; Conlon, M.A.; Bird, A.R.; Topping, D.L.; Le Leu, R.K. Inhibition by resistant starch of red meat-induced promutagenic adducts in mouse colon. Cancer Prev. Res. 2011, 4, 1920-1928.

117. Russell, W.R.; Gratz, Sylvia, S.W.; Duncan, H.; Holtrop, G.; Ince, J.; Scobbie, L.; Duncan, G.; Johnstone, A.M.; Lobley, G.E.; Wallace, R.J.; et al. High-protein, reduced-carbohydrate weight-loss diets promote metabolite profiles likely to be detrimental to colonic health. Am. J. Clin. Nutr. 2011, 93, 1062-1072.

118. Vince, A.; Dawson, A.M.; Park, N.; O'Grady, F. Ammonia production by intestinal bacteria. Gut 1973, 14, 171-177.

119. Magee, E.A.; Richardson, C.J.; Hughes, R.; Cummings, J.H. Contribution of dietary protein to sulfide production in the large intestine: An in vitro and a controlled feeding study in humans. Am. J. Clin. Nutr. 2000, 72, 1488-1494.

120. Noack, J.; Kleessen, B.; Lorenz, A.; Blaut, M. The effect of alimentary polyamine depletion on germ-free and conventional rats. J. Nutr. Biochem. 1996, 7, 560-566. 
121. Richardson, A.J.; McKain, N.; Wallace, R.J. Ammonia production by human faecal bacteria, and the enumeration, isolation and characterization of bacteria capable of growth on peptides and amino acids. BMC Microb. 2013, 13, doi:10.1186/1471-2180-13-6.

122. Caminero, A.; Nistal, E.; Arias, L.; Vivas, S.; Comino, I.; Real, A.; Sousa, C.; Ruiz de Morales, J.M.; Ferrero, M.A.; Rodríguez-Aparicio, L.B.; et al. A gluten metabolism study in healthy individuals shows the presence of faecal glutenasic activity. Eur. J. Nutr. 2012, 51, 293-299.

123. Blachier, F.; Mariotti, F.; Huneau, J.F.; Tome, D. Effects of amino acid-derived luminal metabolites on the colonic epithelium and physiopathological consequences. Amino Acids 2007, $33,547-562$.

124. Brinkworth, G.D.; Noakes, M.; Clifton, P.M.; Bird, A.R. Comparative effects of very low-carbohydrate, high-fat and high-carbohydrate, low-fat weight-loss diets on bowel habit and faecal short-chain fatty acids and bacterial populations. Br. J. Nutr. 2009, 101, 1493-1502.

125. Yamakoshi, J.; Tokutake, S.; Kikuchi, M.; Kubota, Y.; Konishi, H.; Mitsuoka, T. Effect of proanthocyanidin-rich extract from grape seeds on human fecal flora and fecal odor. Microb. Ecol. Health Dis. 2001, 13, 25-31.

126. Goto, K.; Kanaya, S.; Ishigami, T.; Hara, Y. The effects of tea catechins on fecal conditions of elderly residents in a long-term care facility. J. Nutr. Sci. Vitaminol. 1999, 45, 135-141.

127. Macfarlane, G.T.; Allison, C.; Gibson, G.R. Effect of $\mathrm{pH}$ on protease activities in the large intestine. Lett. Appl. Microb. 1988, 7, 161-164.

128. Vince, A.J.; Burridge, S.M. Ammonia production by intestinal bacteria: The effects of lactose, lactulose and glucose. J. Med. Microb. 1980, 13, 177-191.

129. Steck, N.; Mueller, K.; Schemann, M.; Haller, D. Bacterial proteases in IBD and IBS. Gut 2012, $61,1610-1618$.

130. Krogius-Kurikka, L.; Lyra, A.; Malinen, E.; Aarnikunnas, J.; Tuimala, J.; Paulin, L.; Mäkivuokko, H.; Kajander, K.; Palva, A. Microbial community analysis reveals high level phylogenetic alterations in the overall gastrointestinal microbiota of diarrhoea-predominant irritable bowel syndrome sufferers. BMC Gastroenterol. 2009, 9, doi:10.1186/1471-230X-9-95.

131. Etxeberria, U.; Fernández-Quintela, A.; Milagro, F.I.; Aguirre, L.; Martínez, J.A.; Portillo, M.P. Impact of polyphenols and polyphenol-rich dietary sources on gut microbiota composition. J. Agric. Food Chem. 2013, 61, 9517-9533.

132. Joshi, J.R.; Burdman, S.; Lipsky, A.; Yedidia, I. Effects of plant antimicrobial phenolic compounds on virulence of the genus Pectobacterium. Res. Microbiol. 2015, 166, 535-545.

133. Jean-Gilles, D.; Li, L.; Vaidyanathan, V.G.; King, R.; Cho, B.; Worthen, D.R.; Chichester III, C.O.; Seeram, N.P. Inhibitory effects of polyphenol punicalagin on type-II collagen degradation in vitro and inflammation in vivo. Chem.-Biol. Interact. 2013, 205, 90-99.

134. Azuma, T.; Shigeshiro, M.; Kodama, M.; Tanabe, S.; Suzuki, T. Supplemental naringenin prevents intestinal barrier defects and inflammation in colitic mice. J. Nutr. 2013, 143, 827-834.

135. Noda, S.; Tanabe, S.; Suzuki, T. Differential effects of flavonoids on barrier integrity in human intestinal Caco-2 cells. J. Agric. Food Chem. 2012, 60, 4628-4633.

136. Shigeshiro, M.; Tanabe,S.; Suzuki, T. Dietary polyphenols modulate intestinal barrier defects and inflammation in a murine model of colitis. J. Funct. Foods 2013, 5, 949-955. 
137. Carrasco-Pozo, C.; Morales, P.; Gotteland, M. Polyphenols protect the epithelial barrier function of Caco-2 cells exposed to indomethacin through the modulation of occludin and zonula occludens-1 expression. J. Agric. Food Chem. 2013, 61, 5291-5297.

138. Macfarlane, S.; Macfarlane, G.T. Session: Short-chain fatty acids. Regulation of short-chain fatty acid production. Proc. Nutr. Soc. 2003, 62, 67-72.

139. Hughes, R.; Pollock, J.R.A.; Bingham, S. Effect of vegetables, tea, and soy on endogenous $\mathrm{N}$-nitrosation, fecal ammonia, and fecal water genotoxicity during a high red meat diet in humans. Nutr. Cancer 2002, 42, 70-77.

140. Denis, M.-C.; Desjardins, Y.; Furtos, A.; Marcil, V.; Dudonné, S.; Montoudis, A.; Garofalo, C.; Delvin, E.; Marette, A.; Levy, E. Prevention of oxidative stress, inflammation and mitochondrial dysfunction in the intestine by different cranberry phenolic fractions. Clin. Sci. 2015, 128, 197-212.

141. Bernstein, C.N.; Blanchard, J.F.; Kliewer, E.; Wajda, A. Cancer risk in patients with inflammatory bowel disease: A population-based study. Cancer 2001, 91, 854-862.

142. Bhattacharyya, A.; Chattopadhyay, R.; Mitra, S.;Crowe, S.E. Oxidative stress: An essential factor in the pathogenesis of gastrointestinal mucosal diseases. Physiol. Rev. 2014, 94, 329-354.

143. Denis, M.C.; Furtos, A.; Dudonné, S.; Montoudis, A.; Garofalo, C.; Desjardins, Y.; Delvin, E.; Levy, E. Apple peel polyphenols and their beneficial actions on oxidative stress and inflammation. PLoS ONE 2013, 8, e53725.

144. Deiana, M.; Loru, D.; Incani, A.; Rosa, A.; Atzeri, A.; Melis, M.P.; Cabboi, B.; Hollecker, L.; Pinna, M.B.; Argiolas, F.; et al. Wine extracts from Sardinian grape varieties attenuate membrane oxidative damage in Caco-2 cell monolayers. Food Chem. 2012, 134, 2105-2113.

145. Piwowarski, J.P.; Granica, S.; Kiss, A.K. Influence of gut microbiota-derived ellagitannins' metabolites urolithins on pro-inflammatory activities of human neutrophils. Planta Med. 2014, 80, 887-895.

146. Rodríguez-Ramiro, I.; Ramos, S.; Bravo, L.; Goya, L.; Martín, M.T. Procyanidin B2 and a cocoa polyphenolic extract inhibit acrylamide-induced apoptosis in human Caco-2 cells by preventing oxidative stress and activation of JNK pathway. J. Nutr. Biochem. 2011, 22, 1186-1194.

147. Kalaiselvi, P.; Rajashree, K.; Bharathi Priya, L.; Padma, V.V. Cytoprotective effect of epigallocatechin-3-gallate against deoxynivalenol-induced toxicity through anti-oxidative and anti inflammatory mechanisms in HT-29 cells. Food Chem. Toxicol. 2013, 56, 110-118.

148. Mateos-Martín, M.L.; Pérez-Jiménez, J.; Fuguet, E.; Torres, J.L. Profile of urinary and fecal proanthocyanidin metabolites from common cinnamon (Cinnamomum zeylanicum L.) in rats. Mol. Nutr. Food Res. 2012, 56, 671-675.

149. Henning, S.H.; Zhang, Y.; Rontoyanni, V.G.; Huang, J.; Lee, R.-P.; Trang, A.; Nuernberger, G.; Heber, D. Variability in the antioxidant activity of dietary supplements from pomegranate, milk thistle, green tea, grape seed, goji, and acai: Effects of in vitro digestion. J. Agric. Food Chem. 2014, 62, 4313-4321.

150. Fogliano, V.; Corollaro, M.L.; Vitaglione, P.; Napolitano, A.; Ferracane, R.; Travaglia, F.; Arlorio, M.; Costabile, A.; Klinder, A.; Gibson, G. In vitro bioaccessibility and gut biotransformation of polyphenols present in the water-insoluble cocoa fraction. Mol. Nutr. Food Res. 2011, 55, S44-S55. 
151. Goñi, I.; Serrano, J. The intake of dietary fiber from grape seeds modifies the antioxidant status in rat cecum. J. Sci. Food Agric. 2005, 85, 1877-1881.

152. Record, I.R.; McInerney, J.K.; Noakes, M.; Bird, A.R. Chocolate consumption, fecal water antioxidant activity, and hydroxyl radical production. Nutr. Cancer 2003, 47, 131-135.

153. Itzkowitz, S.H.; Yio, X. Inflammation and cancer IV. Colorectal cancer in inflammatory bowel disease: The role of inflammation. Am. J. Physiol. Gastrointest. Liver Physiol. 2004, 287, G7-G17.

154. Rogler G. Gastrointestinal and liver adverse effects of drugs used for treating IBD. Best Pract. Res. Clin. Gastroenterol. 2010, 24, 157-165.

155. Taverniti, V.; Fracassetti, D.; del Bo', C.; Lanti, C.; Minuzzo, M.; Klimis-Zacas, D.; Riso, P.; Guglielmetti, S. Immunomodulatory effect of a wild blueberry anthocyanin-rich extract in human caco-2 intestinal cells. J. Agric. Food Chem. 2014 62, 8346-8351.

156. Nunes, C.; Ferreira, E.; Freitas, V.; Almeida, L.; Barbosa, R.M.; Laranjinha, J. Intestinal anti-inflammatory activity of red wine extract: Unveiling the mechanisms in colonic epithelial cells. Food Funct. 2013, 4, 373-383.

157. Serra, D.; Rufino, A.T.; Mendes, A.F.; Almeida, L.M.; Dinis, T.C.P. Resveratrol modulates cytokine-induced jak/stat activation more efficiently than 5-aminosalicylic acid: An in vitro approach. PLoS ONE 2014, 9, e109048.

158. Miene, C.; Weise, A.; Glei, M. Impact of polyphenol metabolites produced by colonic microbiota on expression of COX-2 and GSTT2 in human colon cells (LT97). Nutr. Cancer 2011, 63, 653-662.

159. Piwowarski, J.P.; Granica, S.; Zwierzyńska, M.; Stefańska, J.; Schopohl, P.; Melzig, M.F.; Kiss, A.K. Role of human gut microbiota metabolism in the anti-inflammatory effect of traditionally used ellagitannin-rich plant materials. J. Ethnopharmacol. 2014, 155, 801-809.

160. Speca, S.; Giusti, I.; Rieder, F.; Latella, G. Cellular and molecular mechanisms of intestinal fibrosis. World J. Gastroent. 2012, 18, 3635-3661.

161. Giménez-Bastida, J.A.; Larrosa, M.; González-Sarrías, A.; Tomás-Barberán, F.; Espín, J.C.; García-Conesa, M.-T. Intestinal ellagitannin metabolites ameliorate cytokine-induced inflammation and associated molecular markers in human colon fibroblasts. J. Agric. Food Chem. 2012, 60, 8866-8876.

162. Sánchez-Fidalgo, S.; Villegas, I.; Aparicio-Soto, M.; Cárdeno, A.; Rosillo, M.T.; González-Benjumea, A.; Marset, A.; López, T.; Maya, I.; Fernández-Bolaños, J.G.; et al. Effects of dietary virgin olive oil polyphenols: Hydroxytyrosyl acetate and 3,4-dihydroxyphenylglycol on DSS-induced acute colitis in mice. J. Nutr. Biochem. 2015, 26, 513-520.

163. Neyrinck, A.M.; van Hée, V.F.; Bindels, L.B.; de Backer, F.; Cani, P.D.; Delzenne, N.M. Polyphenol-rich extract of pomegranate peel alleviates tissue inflammation and hypercholesterolaemia in high-fat diet-induced obese mice: Potential implication of the gut microbiota. Br. J. Nutr. 2013, 109, 802-809.

164. Boussenna, A.; Cholet, J.; Goncalves-Mendes, N.; Joubert-Zakeyh, J.; Fraisse, D.; Vasson, M.P.; Texiera, O.; Felginesa, C. Polyphenol-rich grape pomace extracts protect against dextran sulfate sodium-induced colitis in rats. J. Sci. Food Agric. 2015, in press. 
165. Baumgart, D.C.; Sandborn, W.J. Inflammatory bowel disease: Clinical aspects and established and evolving therapies. Lancet 2007, 369, 1641-1657.

166. Dryden, G.W.; Lam, A.; Beatty, K.; Qazzaz, H.H.; McClain, C.J. A pilot study to evaluate the safety and efficacy of an oral dose of (-)-Epigallocatechin-3-gallate-rich polyphenon $\mathrm{E}$ in patients with mild to moderate ulcerative colitis. Inflamm. Bowel Dis. 2013, 19, 1904-1912.

167. Voiosu, T.; Bengus, A.; Dinu, R.; Voiosu, A.M.; Balanescu, P.; Baicus, C.; Diculescu, M.; Voiosu, R.; Mateescu, B. Rapid fecal calprotectin level assessment and the SIBDQ score can accurately detect active mucosal inflammation in IBD patients in clinical remission: A prospective study. J. Gastrointest. Liver Dis. 2014, 23, 273-278.

168. Burri, E.; Beglinger, C.; von Felten, S.; Lehmann, F.S. Fecal calprotectin and the clinical activity index are both useful to monitor medical treatment in patients with ulcerative colitis. Dig. Dis. Sci. 2014, 60, 485-491.

169. Lehmann, F.S.; Trapani, F.; Fueglistaler, I.; Terracciano, L.M.; von Flüe, M.; Cathomas, G.; Zettl, A.; Benkert, P.; Oertli, D.; Beglinger, C. Clinical and histopathological correlations of fecal calprotectin release in colorectal carcinoma. World J. Gastroenterol. 2014, 20, 4994-4999.

170. Gecse, K.B.; Brandse, J.F.; van Wilpe, S.; Löwenberg, M.; Ponsioen, C.; van den Brink, G.; D'haens, G. Impact of disease location on fecal calprotectin levels in Crohn's disease. Scand. J. Gastroenterol. 2015, 50, 841-847.

171. Fallahi, G.; Motamed, F.; Yousefi, A.; Shafieyoun, A.; Najafi, M.; Khodadad, A.; Farhmand, F.; Ahmadvand, A.; Rezaei, N. The effect of probiotics on fecal calprotectin in patients with cystic fibrosis. Turk. J. Pediatr. 2013, 55, 475-478.

172. Goodrich, K.M.; Fundaro, G.; Griffin, L.E.; Grant, A.; Hulver, M.W.; Ponder, M.A.; Neilson, A.P. Chronic administration of dietary grape seed extract increases colonic expression of gut tight junction protein occludin and reduces fecal calprotectin: A secondary analysis of healthy Wistar Furth rats. Nutr. Res. 2012, 32, 787-794.

173. Biedermann, L.; Mwinyi, J.; Scharl, M.; Frei, P.; Zeitz, J.; Kullak-Ublick, G.A.; Vavricka, S.R.; Fried, M.; Weber, A.; Humpf, H.-U.; et al. Bilberry ingestion improves disease activity in mild to moderate ulcerative colitis-An open pilot study. J. Crohn's Colitis 2013, 7, 271-279.

174. Flint, H.J.; Scott, K.P.; Louis, P.; Duncan, S.H. The role of the gut microbiota in nutrition and health. Nat. Rev. Gastroenterol. Hepatol. 2012, 9, 577-589.

175. Larsen, N.; Vogensen, F.K.; van den Berg, F.W.J.; Nielsen, D.S.; Andreasen, A.S.; Pedersen, B.K.; Al-Soud, W.A.; Sørensen, S.J.; Hansen, L.H.; Jakobsen, M. Gut microbiot in human adults with type 2 diabetes differs from non-diabetic adults. PLOS ONE 2010, 5, e9085.

176. Nicholson J.K.; Holmes, E.; Kinross, J.; Burcelin, R.; Gibson, G.; Jia, W.; Pettersson, S. Host-gut microbiota metabolic interactions. Science 2012, 336, 1262-1267.

177. Hedin, C.; van der Gast, C.J.; Rogers, G.B.; Cuthbertson, L.; McCartney, S.; Stagg, A.J.; Lindsay, J.O.; Whelan, K. Siblings of patients with Crohn's disease exhibit a biologically relevant dysbiosis in mucosal microbial metacommunities. Gut 2015, in press.

178. Wang, J.; Qin, J.; Li, Y.; Cai, Z.; Li, S.; Zhu, J.; Zhang, F.; Liang, S.; Zhang, W.; Guan, Y.; et al. A metagenome-wide association study of gut microbiota in type 2 diabetes. Nature 2012, 490, $55-60$. 
179. Lee, H.C.; Jenner, A.M.; Lowa, C.S.; Lee. Y.K. Effect of tea phenolics and their aromatic fecal bacterial metabolites on intestinal microbiota. Res. Microb. 2006, 157, 876-884.

180. Hütt, P.; Shchepetova, J.; Lõivukene, K.; Kullisaar, T.; Mikelsaar, M. Antagonistic activity of probiotic lactobacilli and bifidobacteria against entero- and uropathogens. J. Appl. Microb. 2006, 100, 1324-1332.

181. Massot-Cladera, M.; Pérez-Berezo, T.; Franch, A.; Castell, M.; Pérez-Cano, F.J. Cocoa modulatory effect on rat faecal microbiota and colonic crosstalk. Arch. Biochem. Biophys. 2012, 527, 105-112.

182. Gibson, G.R.; Beatty, E.R.; Wang, X.; Cummings, J.H. Selective stimulation of bifidobacteria in the human colon by oligofructose and inulin. Gastroenterology 1995, 108, 975-982.

183. Clavel, T.; Fallani, M.; Lepage, P.; Levenez, F.; Mathey, J.; Rochet, V.; Serezat, M.; Sutren, M.; Henderson, G.; Bennetau-Pelissero, C.; et al. Isoflavones and functional foods alter the dominant intestinal microbiota in postmenopausal women. J. Nutr. 2005, 135, 2786-2792.

184. Tzounis, X.; Rodriguez-Mateos, A.; Vulevic, J.; Gibson, G.R.; Kwik-Uribe, C.; Spencer, J.P. Prebiotic evaluation of cocoa-derived flavanols in healthy humans by using a randomized, controlled, double blind, crossover intervention study. Am. J. Clin. Nutr. 2011, 93, 62-72.

185. Clemente-Postigo, M.; Queipo-Ortuño, M.I.; Boto-Ordoñez, M.; Coin-Aragüez, L.; Roca-Rodríguez, M.M.; Delgado-Lista, J.; Cardona, F.; Andres-Lacueva, C.; Tinahones, F.J. Effect of acute and chronic red wine consumption on lipopolysaccharide concentrations. Am. J. Clin. Nutr. 2013, 97, 1053-1061.

186. Vendrame, S.; Guglielmetti, S.; Riso, P.; Arioli, S.; Klimis-Zacas, D.; Porrini, M. Six-week consumption of a wild blueberry powder drink increases bifidobacteria in the human gut. J. Agric. Food Chem. 2011, 59, 12815-12820.

187. Guglielmetti, S.; Fracassetti, D.; Taverniti, V.; del Bo', C.; Vendrame, S.; Klimis-Zacas, D.; Arioli, S.; Riso, P.; Porrini, M. Differential modulation of human intestinal Bifidobacterium populations after consumption of a wild blueberry (Vaccinium angustifolium) Drink. J. Agric. Food Chem. 2013, 61, 8134-8140.

188. Cowan, T.E.; Palmnäs, M.S.A.; Yang, J.; Bomhof, M.R.; Ardell, K.L.; Reimer, R.A.; Vogel, H.J.; Shearer, J. Chronic coffee consumption in the diet-induced obese rat: Impact on gut microbiota and serum metabolomics. J. Nutr. Biochem. 2014, 25, 489-495.

189. Duncan, S.H.; Lobley, G.E.; Holtrop, G.; Ince, J.; Johnstone, A.M.; Louis, P.; Flint, H.J. Human colonic microbiota associated with diet, obesity and weight loss. Int. J. Obes. 2008, 32, 1720-1724.

190. Kotlowski, R.; Bernstein, C.N.; Sepehri, S.; Krause, D.O. High prevalence of Escherichia coli belonging to the B2+D phylogenetic group in inflammatory bowel disease. Gut 2007, 56, 669-675.

191. Bustos, I.; García-Cayuela, T.; Hernández-Ledesma, B.; Peláez, C.; Requena, T.; Martínez-Cuesta, M.C. Effect of flavan-3-ols on the adhesion of potential probiotic lactobacilli to intestinal cells. J. Agric. Food Chem. 2012, 60, 9082-9088.

(C) 2015 by the authors; licensee MDPI, Basel, Switzerland. This article is an open access article distributed under the terms and conditions of the Creative Commons Attribution license (http://creativecommons.org/licenses/by/4.0/). 